\title{
Genome-wide association meta-analysis for early age-related macular degeneration highlights novel loci and insights for advanced disease
}

Thomas W. Winkler ${ }^{1 *+}$ (D), Felix Grassmann ${ }^{2,3,4 \dagger}$, Caroline Brandl ${ }^{1,2,5}$, Christina Kiel ${ }^{2}$, Felix Günther ${ }^{1,6}$, Tobias Strunz ${ }^{2}$, Lorraine Weidner ${ }^{1}$, Martina E. Zimmermann ${ }^{1}$, Christina A. Korb ${ }^{7}$, Alicia Poplawski ${ }^{8}$, Alexander K. Schuster ${ }^{7}$, Martina Müller-Nurasyid ${ }^{8,9,10,11}$, Annette Peters ${ }^{12,13}$, Franziska G. Rauscher ${ }^{14,15}$, Tobias Elze ${ }^{14,16}$, Katrin Horn ${ }^{14,15}$, Markus Scholz ${ }^{14,15}$, Marisa Cañadas-Garre ${ }^{17}$, Amy Jayne McKnight ${ }^{17}$, Nicola Quinn ${ }^{17}$, Ruth E. Hogg ${ }^{17}$, Helmut Küchenhoff ${ }^{6}$, Iris M. Heid ${ }^{1 \dagger}$, Klaus J. Stark ${ }^{1+}$ and Bernhard H. F. Weber ${ }^{2,18+}$

\section{Abstract}

Background: Advanced age-related macular degeneration (AMD) is a leading cause of blindness. While around half of the genetic contribution to advanced AMD has been uncovered, little is known about the genetic architecture of early AMD.

Methods: To identify genetic factors for early AMD, we conducted a genome-wide association study (GWAS) meta-analysis (14,034 cases, 91,214 controls, 11 sources of data including the International AMD Genomics Consortium, IAMDGC, and UK Biobank, UKBB). We ascertained early AMD via color fundus photographs by manual grading for 10 sources and via an automated machine learning approach for > 170,000 photographs from UKBB. We searched for early AMD loci via GWAS and via a candidate approach based on 14 previously suggested early AMD variants.

(Continued on next page)

\footnotetext{
* Correspondence: thomas.winkler@klinik.uni-regensburg.de

Thomas W Winkler and Felix Grassmann contributed equally

'Iris M Heid, Klaus J Stark and Bernhard HF Weber jointly supervised this work

'Department of Genetic Epidemiology, University of Regensburg, Regensburg, Germany

Full list of author information is available at the end of the article
}

(c) The Author(s). 2020 Open Access This article is licensed under a Creative Commons Attribution 4.0 International License, which permits use, sharing, adaptation, distribution and reproduction in any medium or format, as long as you give appropriate credit to the original author(s) and the source, provide a link to the Creative Commons licence, and indicate if changes were made. The images or other third party material in this article are included in the article's Creative Commons licence, unless indicated otherwise in a credit line to the material. If material is not included in the article's Creative Commons licence and your intended use is not permitted by statutory regulation or exceeds the permitted use, you will need to obtain permission directly from the copyright holder. To view a copy of this licence, visit http://creativecommons.org/licenses/by/4.0/ The Creative Commons Public Domain Dedication waiver (http://creativecommons.org/publicdomain/zero/1.0/) applies to the data made available in this article, unless otherwise stated in a credit line to the data. 
(Continued from previous page)

Results: Altogether, we identified 10 independent loci with statistical significance for early AMD: (i) 8 from our GWAS with genome-wide significance $\left(P<5 \times 10^{-8}\right)$, (ii) one previously suggested locus with experiment-wise significance $(P<0.05 /$ 14) in our non-overlapping data and with genome-wide significance when combining the reported and our nonoverlapping data (together 17,539 cases, 105,395 controls), and (iii) one further previously suggested locus with experimentwise significance in our non-overlapping data. Of these 10 identified loci, 8 were novel and 2 known for early AMD. Most of the 10 loci overlapped with known advanced AMD loci (near ARMS2/HTRA1, CFH, C2, C3, CETP, TNFRSF10A, VEGFA, APOE), except two that have not yet been identified with statistical significance for any AMD. Among the 17 genes within these two loci, in-silico functional annotation suggested CD46 and TYR as the most likely responsible genes. Presence or absence of an early AMD effect distinguished the known pathways of advanced AMD genetics (complement/lipid pathways versus extracellular matrix metabolism).

Conclusions: Our GWAS on early AMD identified novel loci, highlighted shared and distinct genetics between early and advanced AMD and provides insights into AMD etiology. Our data provide a resource comparable in size to the existing IAMDGC data on advanced AMD genetics enabling a joint view. The biological relevance of this joint view is underscored by the ability of early AMD effects to differentiate the major pathways for advanced AMD.

Keywords: Genome-wide association study (GWAS), Meta-analysis, Age-related macular degeneration (AMD), Early AMD, CD46, TYR, International AMD genomics consortium (IAMDGC), UK biobank (UKBB), Machine-learning, Automated phenotyping

\section{Background}

Age-related macular degeneration (AMD) is the leading cause of irreversible central vision impairment in industrialized countries. Advanced AMD presents as geographic atrophy (GA) and/or neovascular (NV) complications [1]. Typically, advanced AMD is preceded by clinically asymptomatic and thus often unrecognized early disease stages. Early AMD is characterised by differently sized yellowish accumulations of extracellular material between Bruch's membrane and retinal pigment epithelium (RPE) or between RPE and the photoreceptors (drusen or subretinal drusenoid deposits, respectively). Other features of early AMD are RPE abnormalities, including depigmentation or increased amount of pigment [1].

Early and advanced AMD can be documented by color fundus imaging of the central retina and/or other multimodal imaging approaches including optical coherence tomography (OCT) [1-3]. While the definition of advanced AMD is reasonably homogeneous across clinical and epidemiological studies, the classification of early AMD is more variable and different studies traditionally apply differing classification systems $[4,5]$.

Epidemiological studies show that high age is the strongest risk factor for early and advanced AMD onset as well as progression [1,6-8]. A robust genetic influence was shown for advanced AMD [1, 9-11] with 34 distinct loci at genome-wide significance in a large genome-wide association study (GWAS) for advanced AMD [9]. The genes underneath these advanced AMD loci were found to be enriched for genes in the alternative complement pathway, HDL transport, and extracellular matrix organization and assembly [9].
Exploring the genetics of early AMD offers the potential to understand the mechanisms of early disease processes, but also for the development to advanced AMD when comparing genetic effect sizes for early and advanced stages. Yet there have been few published GWAS searches for early AMD. One meta-analysis on 4089 early AMD patients and 20,453 control persons reported two loci with genomewide significance, both being well known from advanced AMD, the CFH and the ARMS2/HTRA1 locus [12].

We have thus set out to gather GWAS data for early AMD from 11 sources including own study data, data from the International AMD Genomics Consortium (IAMDGC), dbGaP and UK Biobank to conduct the largest GWAS meta-analysis on early AMD to date.

\section{Methods \\ GWAS data from 11 sources}

We included 11 sources of data with GWAS data and color fundus photography for early AMD phenotyping (Table S1). Our studies were primarily population-based cohort studies, where the baseline survey data were used for this analysis from studies of the authors (GHS, LIFE, NICOLA, KORA, AugUR) as well as for publicly available studies from dbGaP (ARIC, CHS, WHI; accession numbers: phs000090.v5.p1, phs000287.v6.p1, phs000746.v2.p3). We also included data from UK Biobank for participants from baseline and additional participants from the follow-up survey, since the color fundus photography program had started only after the main study onset (application number \#33999). The studies captured an age range from 25 to 100 years of age (mean age from 47.5 years to 77.2 years across the 10 population-based studies, AugUR with the very old individuals range from 70.3 years to 95 years). About $50 \%$ 
of the study participants in each study were male (except for the Women's Health Initiative, WHI), and all demonstrated European ancestry. All studies (except GHS-1, GHS-2 and Life-Adult) excluded any person with at least 2nd degree relationship (Table S1). For each of these crosssectional data sets, participants with at least one eye gradable for AMD (see below) and with existing GWAS data were eligible for our analysis. We excluded participants with advanced AMD. We used participants with ascertained early AMD as cases and participants being ascertained for not having any signs of AMD as controls $(n=$ 7363 cases, 73,358 controls across these population-based studies). Case-control data were also included from IAMD GC (http://amdgenetics.org/). The early AMD GWAS from IAMDGC is based on 24,527 individual participant data from 26 sources [9]. This data includes 17,856 participants with no AMD and 6671 participants with early AMD (excluding the 16,144 participants with advanced AMD). The cases and controls from IAMDGC were 16 to 102 years of age (mean age $=71.7$ years). For all of these participants, DNA samples had been gathered and genotyped centrally (see below) [9].

\section{Genotyping and imputation}

All population-based studies were genotyped, quality controlled and imputed using similar chip platforms and imputation approaches (Table S2). As the imputation backbone, the 1000 Genomes Phase 1 or Phase 3 reference panel was applied [13], except GHS was imputed based on the Haplotype Reference Consortium (HRC) [14] and UK Biobank was imputed based on HRC and the UK10K haplotype resource [15]. Details on the UK Biobank genotypic resource are described elsewhere [16]. For the IAMDGC case-control data, DNA samples had been gathered across all participants and genotyped on an Illumina HumanCoreExome array and quality controlled centrally. Genotype quality control and imputation to the 1000 Genomes phase 1 version 3 reference panel (>12 million variants) were conducted centrally. Details on the IAMDGC data were described in detail by Fritsche et al. [9].

\section{Phenotyping}

Across all studies included into this analysis, early AMD and the unaffected status was ascertained by color fundus photography. For participants from AugUR and LIFE, "early AMD" was classified according to the Three Continent Consortium (3CC) Severity Scale [4], which separates "mild early" from "moderate" and "severe early" AMD stages depending on drusen size, drusen area, or the presence of pigmentary abnormalities [4]. For the analysis, we collapsed any of these "early" AMD stages into the definition of "early AMD". However, the 3CC Severity Scale was not available for the other studies. In these, similar early AMD classifications, considering drusen size or area and presence of pigmentary abnormalities, were used (Table S1): For participants from GHS, the Rotterdam Eye Study classification was applied [17]. For participants from NICOLA, the Beckman Clinical Classification was utilized [18]. Participants from the KORA study were classified as "early AMD" based on the AREDS-9 step classification scheme and we defined "early AMD" for this analysis by AREDS-9 steps $2-8$ [19]. The ascertainment of IAMDGC study participants is described in detail elsewhere and covers various classification systems [9]. Of note, LIFE and NICOLA phenotyping incorporated OCT information additional to the information from color fundus imaging (Table S1). For UK Biobank participants, color fundus images were received (application number 33999); there was no existing AMD classification available in UK Biobank (see below). The AMD status of a person was derived based on the AMD status of the eye with the more severe AMD stage ("worse eye") when both eyes were gradable, and as the grade of the one available eye otherwise. Eyes were regarded as gradable, if at least one image of the eye fulfilled defined quality criteria allowing for the assessment of AMD (bright image, good color contrast, full macular region captured on images). Images were excluded from AMD grading if they revealed obscuring lesions (e.g. cataract) or lesions considered to be the result of a competing retinal disease (such as advanced diabetic retinopathy, high myopia, trauma, congenital diseases, or photocoagulation unrelated to choroidal neovascularization). Details for IAMDGC are described previously [9]. Persons with gradable images for at least one eye were included in this analysis. Persons with advanced AMD defined as presence of neovascularization or geographic atrophy in at least one eye were excluded for the main GWAS on early AMD.

\section{Automated classification of early AMD in UK biobank}

To obtain early AMD phenotype data for UK Biobank participants, we used a pre-trained algorithm for automated AMD classification based on an ensemble of convolutional neural networks [20]. In the UKBB baseline data, fundus images were available for 135,500 eyes of 68,400 individuals with at least one image. Among the additional 38,712 images of 19,501 individuals in the follow-up, there were 17,198 individuals without any image from baseline. For each image (eye) at baseline and follow-up, we predicted the AMD stage on the AREDS-9 step severity scale using the automated AMD classification. We defined a person-specific AMD stage at baseline and follow-up based on the worse eye. Eyes that were classified as ungradable were treated as missing data and, if diagnosis was available for only one eye, the person-specific AMD stage was based on the 
classification of the single eye. If we obtained an automated disease classification to an AMD stage (i.e. not "ungradable" for both eyes) at baseline and follow-up, we used the follow-up disease stage (and follow-up age) in the association analysis. By this, we obtained an automated AMD classification for 70,349 individuals (2161 advanced AMD, 3835 early AMD, 64,353 unaffected). Individuals with advanced AMD were excluded from this analysis. Finally, we yielded 57,802 unrelated individuals of European ancestry with valid GWAS data that had either early AMD or were free of any AMD (3105 cases, 54,697 controls). We evaluated the performance of the automated disease classification by selecting 2013 individuals (4026 fundus images) for manual classification based on the 3CC Severity Scale. Details are described elsewhere [21]. We found reasonable agreement between the automated and the manual classification for the four categories of "no AMD", "early AMD", "advanced AMD" and "ungradable" (concordance $=79.5 \%$, Cohen's kappa $\kappa=0.61$, kappa with list-wise exclusion of ungradable individuals $k=0.47$ [22]). We found 305 of the 2013 individuals to be ungradable by the automated approach (i.e. 15.2\%), including 257 individuals that have also been ungradable manually (i.e. truly bad image quality for both eyes) and 48 individuals that had been manually gradable, but not with the automated approach (i.e. 2.4\%). Further details and further comparisons are provided in the Supplementary Note and Tables S3-S4.

\section{Study-specific association analyses}

Study-specific logistic regression analyses (early AMD cases versus controls, excluding advanced AMD cases) were applied by study partners (in Regensburg, Leipzig, Mainz, Belfast) using an additive genotype model and according to a pre-defined analysis plan. All publicly available data from dbGAP (studies ARIC, CHS and WHI) and UK Biobank as well as IAMDGC data was analyzed in Regensburg. All studies inferred the association of each genetic variant with early AMD using a Wald test statistic as implemented in RVTESTS [23]. Age and two principal components (to adjust for population stratification) were included as covariates in the regression models. We conducted sensitivity analyses to evaluate the impact of additionally including sex and 10 principal components in the regression models on the example of the two largest data sets, the IAMDGC and the UKBB data. The IAMDGC analyses were further adjusted for DNA source as done previously [9]. For the IAMDGC data that stemmed from 26 sources, we conducted a sensitivity analysis additionally adjusting for source membership according to previous work highlighting slight differences in effect estimates [24]; we found the same results.
Quality control of study-specific aggregated data GWAS summary statistics for all data sources were processed through a standardized quality-control (QC) pipeline [25]. This involved QC checks on file completeness, range of test statistics, allele frequencies, population stratification as well as filtering on low quality data. We excluded variants with low minor allele count $(\mathrm{MAC}<10$, calculated as $\mathrm{MAC}=2 * \mathrm{~N}_{\text {eff }}$ "MAF, with $\mathrm{N}_{\text {eff }}$ being the effective sample size, $\mathrm{N}_{\text {eff }}=4 \mathrm{~N}_{\text {Cases }}{ }^{*} \mathrm{~N}_{\text {Controls }} /\left(\mathrm{N}_{\text {Cases }}+\mathrm{N}_{\text {Con- }}\right.$ trols) and MAF being the minor allele frequency), low imputation quality $(\mathrm{rsq}<0.4)$ or large standard error of the estimated genetic effect $(\mathrm{SE}>10)$. Genomic control (GC) correction was applied to each GWAS result to correct for population stratification within each study [26]. The estimation of the GC inflation factor was based on variants outside of the 34 known advanced AMD regions (excluding all variants within $<5 \mathrm{Mb}$ base positions to any of the 34 known advanced AMD lead variants). The GC factors ranged from 1.00 to 1.04 (Table S2). We transferred all variant identifiers to unique variant names consisting of chromosomal, base position (hg19) and allele codes in (e.g. "3:12345:A_C", allele codes in ASCII ascending order).

\section{GWAS meta-analysis across the 11 sources of data for early AMD genetics}

For signal detection and effect quantification, studyspecific genetic effects were combined using an inversevariance weighted fixed effect meta-analysis method as implemented in METAL [27]. We performed additional QC on meta-analysis results: We only included variants for identification that were available (i) in at least two of the 11 data sources with a total effective sample size of more than 5000 individuals $\left(\mathrm{N}_{\text {eff }}>5000\right)$ and (ii) for chromosome and position annotation in dbSNP (hg19). A conservative second GC correction (again focusing on variants outside the known advanced AMD regions) was applied to the meta-analysis result, in order to correct for potential population stratification across studies [26]. The GC lambda factor of the meta-analysis was 1.01 .

\section{Genome-wide search for early AMD variants, variant selection, and locus definition}

In our first approach, we conducted a genome-wide search for variants associated with early AMD and judged at a genome-wide significance level $(P<5.0 \times$ $\left.10^{-8}\right)$. Variants identified by this GWAS approach were deemed as established with genome-wide significance in our meta-analysis (tier 1). To evaluate the robustness of any novel genome-wide significant AMD locus, we performed leave-one-out (LOO) meta-analyses. We also evaluated heterogeneity between study-specific genetic effect estimates for selected variants using the $\mathrm{I}^{2}$ measures [28-30]. 
We combined genome-wide significant variants $(\mathrm{P}<$ $5.0 \times 10^{-8}$ ) into independent loci by using a locus definition similar to what was done previously [9]: the most significant variant was selected genome-wide, all variants were extracted that were correlated with this lead variant $\left(\mathrm{r}^{2}>0.5\right.$, using IAMDGC controls as reference) and a further $500 \mathrm{~kb}$ were added to both sides. All variants overlapping the sodefined locus were assigned to the respective locus. We repeated the procedure until no further genome-wide significant variants were detected. Genes overlapping the sodefined loci were used for biological follow-up analyses (gene region defined from start to end). To identify independent secondary signals at any novel AMD locus, approximate conditional analyses were conducted based on meta-analysis summary statistics using GCTA [31].

\section{Candidate approach}

Additionally to the genome-wide search in our metaanalysis of 14,034 cases and 91,214 controls, we adopted a candidate approach based on the 14 reported suggestive variants by Holliday et al. ( $P$-values from $8.9 \times 10^{-6}$ to $1.1 \times 10^{-6}$ in their meta-analysis, 4089 cases and 20 , 453 controls) [12]. For this, we analyzed our data without the studies that overlapped with the previously reported data (i.e. removing ARIC, CHS; yielding 13,450 cases and 84,942 controls). We also combined the data from Holliday et al. with this non-overlapping part of our data where possible (i.e. the 14 reported variants) by an inverse-variance weighted meta-analysis (altogether 17,539 cases, 105,395 controls). We judged these 14 variants' association at experiment-wise significance $(P<$ $0.05 / 14$ ) in our non-overlapping data and at genomewide significance $\left(\mathrm{P}_{\text {Combined }}<5.0 \times 10^{-8}\right)$ in the combined analysis. We considered a candidate-based selected variant as established with both experiment-wise significance $(\mathrm{P}<0.05 / 14$ in our non-overlapping data) and with genome-wide significance $\left(\mathrm{P}_{\text {Combined }}<5.0 \times\right.$ $10^{-8}$ in the combined data) (tier 2), or as established with experiment-wise significance $(\mathrm{P}<0.05 / 14$ in our non-overlapping data), but without established genomewide significance $\left(\mathrm{P}_{\text {Combined }} \geq 5.0 \times 10^{-8}\right)($ tier 3$)$.

\section{Gene prioritization at newly identified AMD loci}

To prioritize genes and variants at the newly identified AMD loci, we conducted a range of statistical and functional follow-up analyses. The following criteria were used: (1) Statistical evidence; we computed the posterior probability of each variant using Z-scores and derived 95\% credible intervals for each locus [32]. The method assumes a single causal signal per locus. (2) Variant effect predictor (VEP) to explore whether any of the credible variants was located in a relevant regulatory gene region [33]. (3) eQTL analysis: We downloaded expression summary statistics for the candidate genes in retina from the EyeGEx database [34] and for 44 other tissues from the GTEx database [35] (both available at www.gtexportal.org/home/ datasets) and evaluated whether any of the credible variants showed significant effects on expression levels in the aggregated data. For each significant eQTL in EyeGEx, we conducted colocalization analyses using eCAVIAR [36] to evaluate whether the observed early AMD association signal colocalized with the variants' association with gene expression. (4) Retinal expression: We queried the EyeIntegration database to evaluate genes in the relevant loci for expression in fetal or adult retina or RPE cells [37]. (5) Animal model: We queried the Mouse Genome Informatics (MGI) database (www.informatics.jax.org) for each gene in the relevant loci for relevant eye phenotypes in mice [38]. (6) Human phenotype: The Online Mendelian Inheritance in Man (OMIM) $)^{\circ}$ database was queried for human eye phenotypes (McKusick-Nathans Institute of Genetic Medicine, Johns Hopkins University, Baltimore, MD, queried 07/11/2019, www.omim.org).

\section{Phenome-wide association study for newly identified AMD loci}

We used 82 other traits and queried reported genomewide significant $\left(P<5.0 \times 10^{-8}\right)$ lead variants and proxies $\left(\mathrm{r}^{2}>0.5\right)$ for any of these traits for overlap with genes underneath our novel loci as done previously [39]. For this, we used GWAS summary results that were previously aggregated from GWAS catalogue [40], GWAS central [41] and literature search.

For the novel early AMD lead variants, we further evaluated their association with 118 non-binary and 660 binary traits from the UK Biobank [42]. The Phenome-wide association study (PheWAS) web browser "GeneATLAS" (www. geneatlas.roslin.ed.ac.uk) was used for the UK Biobank lookup. For each variant, association $P$ values were corrected for the testing of multiple traits by the Benjamini-Hochberg false-discovery-rate (FDR) method [43].

\section{Interaction analyses}

For the novel early AMD effects and for the 34 known advanced AMD lead variants [9], we investigated whether age modulated early AMD effects by analyzing variant $\mathrm{x}$ age interaction in seven data sources for which we had individual participant data available in Regensburg (ARIC, CHS, WHI, IAMDGC, UKBB, AugUR and KORA). For each source, we applied logistic regression and included a variant $\mathrm{x}$ AGE interaction term in the model (in addition to the covariates used in the main analysis). We conducted meta-analysis across the seven sources to obtain pooled variant $\mathrm{x}$ age interaction effects and applied a Wald test to test for significant interaction (at a Bonferroni-corrected alpha-level). For the novel early AMD effects, we further investigated whether age modulated advanced AMD effects by evaluating 
publically available data from IAMDGC [44]. Finally, we investigated whether a novel early AMD lead variant modulated any of the effects of the 34 known AMD variants on advanced AMD [9]. We used the IAMDGC data and applied one logistic regression model for each pair of known advanced AMD variants and novel early AMD variants including the two respective variants and their interaction (and the same other covariates as before).

\section{Comparison of genetic effects on early and advanced stage AMD}

We estimated the genetic correlation between early and advanced AMD by utilizing the LDSC tool [45] with the GWAS summary statistics for early and advanced AMD (from the current meta-analysis and the IAMDGC [9], respectively). We used pre-calculated LD scores for European ancestry (https://data.broadinstitute.org/alkesgroup/ LDSCORE/eur_w_ld_chr.tar.bz2). We further compared genetic effect sizes between early and advanced AMD for the novel early AMD lead variants and for the 34 known advanced AMD lead variants [9]. For this, we queried the novel early AMD lead variants in the IAMDGC GWAS for advanced AMD [9] and (vice-versa) queried the 34 known advanced AMD lead variants [9] in the early AMD metaanalysis results. We compared effect sizes in a scatter plot and clustered the lead variants by their nominal significant association on advanced and/or early AMD. We classify different types of loci in a similar fashion as done previously for adiposity trait genetics [46]: (1) "advanced-and-early" AMD loci $\left(\mathrm{P}_{\text {early }}<0.05, \mathrm{P}_{\mathrm{adv}}<0.05\right)$, (2) "advanced-only" AMD loci $\left(\mathrm{P}_{\text {early }} \geq 0.05, \mathrm{P}_{\mathrm{adv}}<0.05\right)$, (3) "early-only" AMD loci $\left(\mathrm{P}_{\text {early }}<0.05, \mathrm{P}_{\mathrm{adv}} \geq 0.05\right)$.

\section{Pathway analysis}

To evaluate whether "advanced-and-early" AMD loci versus "advanced-only" AMD loci distinguished the major known pathways for advanced AMD, we performed pathway enrichment analysis separately for these two classes. We used the genes in the gene prioritization for all advanced AMD loci as previously described [9], derived the gene prioritization score and selected the best scored gene in each locus (two genes in the case of ties). We then separated the gene list according to the class of the respective locus, and performed pathway enrichment analysis via Enrichr [47] with default settings searching Reactome's cell signaling pathway database 2016 ( $n=1530$ pathways). $P$ values were corrected for multiple testing with BenjaminiHochberg procedure [43].

\section{Results}

Eight genome-wide significant loci from a GWAS on early AMD

We conducted a meta-analysis of genotyped and imputed data from 11 sources (14,034 early AMD cases,
91,214 controls; 11,702,853 variants; for study-specific genotyping, analysis and QC, see Tables S1-S2). For all participants, early AMD or control status (i.e. no early nor late AMD) was ascertained via color fundus photographs (Table S1). This included automated machinelearning based AMD classification of UK Biobank fundus images (application number 33999; 56,699 individuals from baseline, 13,650 additional individuals from follow-up) [16, 20]. Based on logistic regression association analysis in each of the 11 data sets meta-analyzed via fixed effect model, we identified eight distinct loci with genome-wide significance (tier $1 ; P=1.3 \times 10^{-116}$ to $4.7 \times 10^{-8}$, Fig. 1, Table 1; "locus" defined by the lead variant and proxies, $\left.\mathrm{r}^{2} \geq 0.5,+/-500 \mathrm{~kb}\right)$. Six of these loci were novel for early AMD; two loci had been identified for early AMD previously [12].

Most of the eight loci overlap with known loci for advanced AMD [9] (CFH, ARMS2/HTRA1, C2, C3, CETP, VEGFA, TNFRSF10A), except one which has not been identified in early or advanced AMD GWAS before $(P=$ $4.7 \times 10^{-8}$, lead variant rs4844620, near CD46, Figure $\mathrm{S} 1$ ). This novel locus around rs4844620 (near CD46) is independent of known (advanced) AMD loci: rs4844620 is (i) $\sim 500 \mathrm{~K}$ base positions distant from the recently reported AMD locus near C4BPA/CD55 [11] and uncorrelated to the reported $C 4 B P A / C D 55$ lead variant rs11120691 $\left(\mathrm{r}^{2}<0.01\right)$ and (ii) $>10$ million base positions distant from the $C F H$ locus and uncorrelated to any of the eight reported $C F H$ locus variants, $\left.\mathrm{r}^{2}<0.01\right)$.

Taken together, we identified eight loci for early AMD, two known for early AMD and six novel, including one novel locus (near CD46) for any AMD with genome-wide significance.

\section{Two further significant loci from a candidate-based approach of 14 variants}

Subsequently, we applied a candidate-based approach by investigating the 14 variants reported as suggestive by the previous GWAS for early AMD (4089 early AMD cases, 20,453 controls; reported $\mathrm{P}$ between $1.1 \times 10^{-6}$ and $8.9 \times 10^{-6}$ ) [12]. For this, we re-analyzed our data excluding the overlap with the previous GWAS (i.e. excluding ARIC, CHS study, yielding 13,450 early AMD cases and 84,942 controls) and also combined the reported and our non-overlapping data in an inversevariance weighted meta-analysis where possible (i.e. for the 14 reported variants; altogether 17,539 cases, 105, 395 controls). Among the 14 variants, we found four significant variants for early AMD (Table 2): (i) two "tier 2" variants (near PVRL2 and CD46) with experiment-wise significance $(P<0.05 / 14)$ in our non-overlapping data and with genome-wide significance $\left(\mathrm{P}_{\text {Combined }}<5.0 \times\right.$ $10^{-8}$ ) in the combined analysis, and (ii) two additional "tier 3" variants (near APOE/TOMM4O and TYR) with 


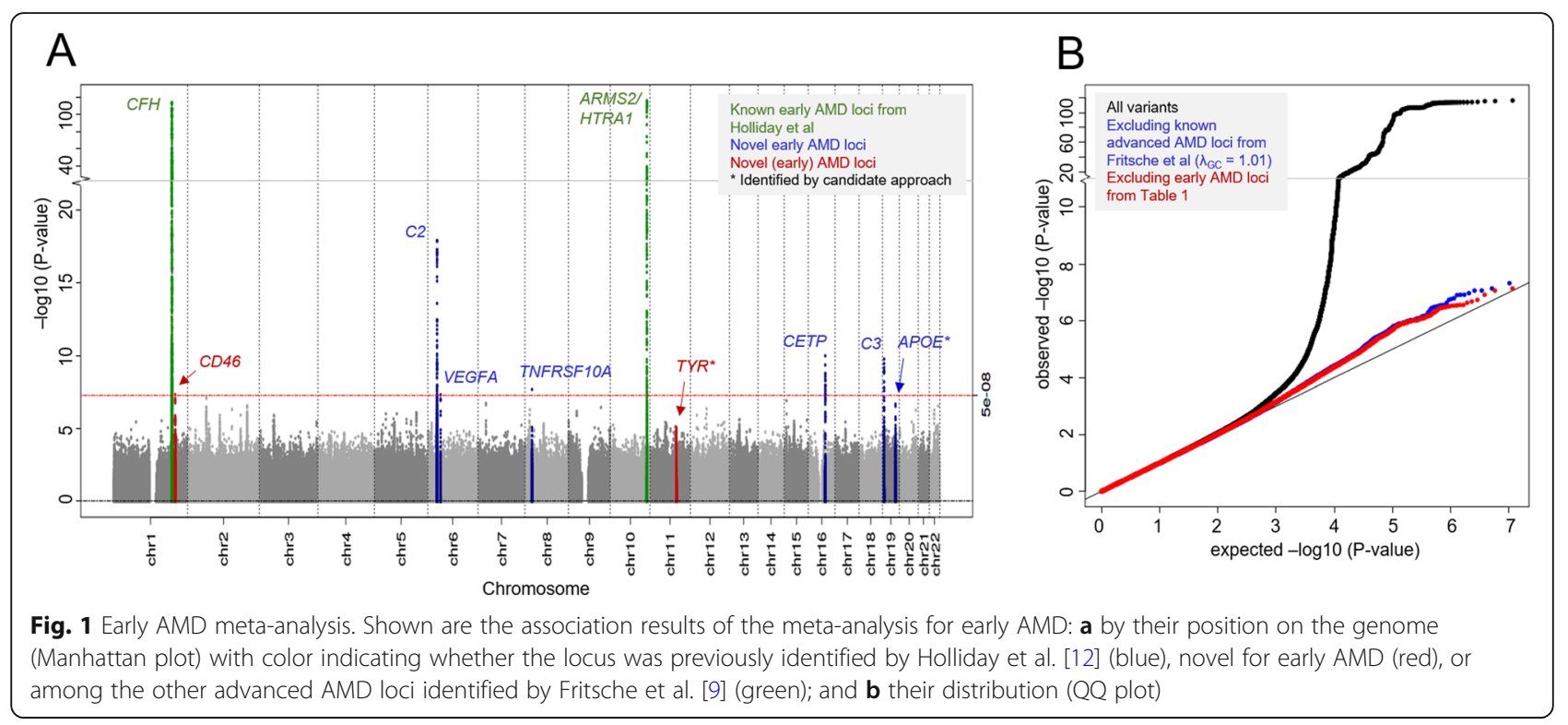

experiment-wise significance $(\mathrm{P}<0.05 / 14)$ in our nonoverlapping data.

We compared the evidence from the Bonferronicorrected analysis judged at experiment-wise significance and the combined analysis judged at genome-wide significance. Both "tier 3" variants showed combined Pvalues close to the genome-wide significant threshold $\left(\mathrm{P}_{\text {Combined }}=9.0 \times 10^{-8}\right.$ and $1.7 \times 10^{-7}$, for the $A P O E /$ TOMM40 and the TYR variant, respectively), while the other 10 of the 14 variants showed combined P-values far away from significance $\left(\mathrm{P}_{\text {combined }}\right.$ from $4.8 \times 10^{-3}$ to 0.74). Therefore, the Bonferroni-corrected analysis and the combined analysis yielded similar evidence and separated the 14 suggested variants into four variants with a positive finding (near CD46, PVRL2, APOE/TOMM4O and TYR ) from the 10 variants with no finding $(P>0.05$ in our non-overlapping data, Table 2).

Two of the four identified variants were correlated: the tier3-identified variant rs2075650 near APOE/TOMM40 was located in the same locus as the tier2-identified variant rs6857 near PVRL2 $\left(\mathrm{r}^{2}=0.75\right.$ to rs2075650) and thus counted as one locus (near APOE/TOMM40/PVRL2). The tier2-identified variant rs1967689 near CD46 was located in one of the eight loci identified by our GWAS $\left(r^{2}=0.77\right.$ to our GWAS lead variant $\left.\mathrm{rs} 4844620\right)$. The two other loci (near APOE/TOMM40/PVRL2 and TYR) were identified in addition to our GWAS. These two loci were identified here for the first time with statistical significance as loci for early AMD: one locus known for advanced AMD (near $A P O E$ ) and one identified here for

Table 1 Genome-wide search for early AMD association

\begin{tabular}{|c|c|c|c|c|c|c|c|c|c|c|c|c|}
\hline Rs identifier & chr:pos [hg19] & EA & $\mathrm{OA}$ & EAF & $\log O R$ & SE & OR & $P$ & $\mathrm{~N}$ cases & $\mathrm{N}$ controls & $\begin{array}{l}\text { Known advanced } \\
\text { AMD locus (Fritsche et al.) }\end{array}$ & Locus name \\
\hline \multicolumn{13}{|c|}{ Novel early AMD loci: } \\
\hline rs4844620 & $1: 207980901$ & $g$ & a & 0.79 & 0.095 & 0.017 & 1.10 & 4.7E-08 & 14,031 & 91,179 & no & CD46 \\
\hline rs547154 & 6:31910938 & $g$ & $t$ & 0.91 & 0.218 & 0.025 & 1.24 & $1.3 \mathrm{E}-18$ & 14,027 & 91,137 & yes & $C 2$ \\
\hline rs943080 & $6: 43826627$ & $t$ & C & 0.51 & 0.080 & 0.015 & 1.08 & 4.7E-08 & 13,220 & 85,747 & yes & VEGFA \\
\hline rs13278062 & $8: 23082971$ & $t$ & $g$ & 0.52 & 0.080 & 0.014 & 1.08 & 2.0E-08 & 13,644 & 85,908 & yes & TNFRSF10A \\
\hline rs5817082 & $16: 56997349$ & C & $\mathrm{ca}$ & 0.26 & 0.108 & 0.017 & 1.11 & $1.0 \mathrm{E}-10$ & 12,599 & 81,863 & yes & CETP \\
\hline rs11569415 & $19: 6716279$ & a & $g$ & 0.21 & 0.116 & 0.018 & 1.12 & 1.7E-10 & 13,115 & 83,117 & yes & C3 \\
\hline \multicolumn{13}{|c|}{ Known early AMD loci: } \\
\hline rs4658046 & $1: 196670757$ & C & $t$ & 0.39 & 0.321 & 0.014 & 1.38 & $2.9 E-114$ & 14,034 & 91,201 & yes & $\mathrm{CFH}$ \\
\hline rs3750847 & $10: 124215421$ & $\mathrm{t}$ & $c$ & 0.22 & 0.384 & 0.017 & 1.47 & $1.3 \mathrm{E}-116$ & 14,025 & 91,171 & yes & ARMS2/HTRA1 \\
\hline
\end{tabular}

$E A$ effect allele, $O A$ other allele, EAF effect allele frequency, $\log O R \log$ odds ratio, SE standard error of logOR; OR odds ratio, $P$ double GC corrected early association $P$ value from the meta-analysis

The table shows the eight genome-wide significant $\left(P<5.0 \times 10^{-8}\right)$ lead variants from the early AMD meta-analysis. The second last column indicates whether the locus was identified by Fritsche et al. for advanced AMD [9] 


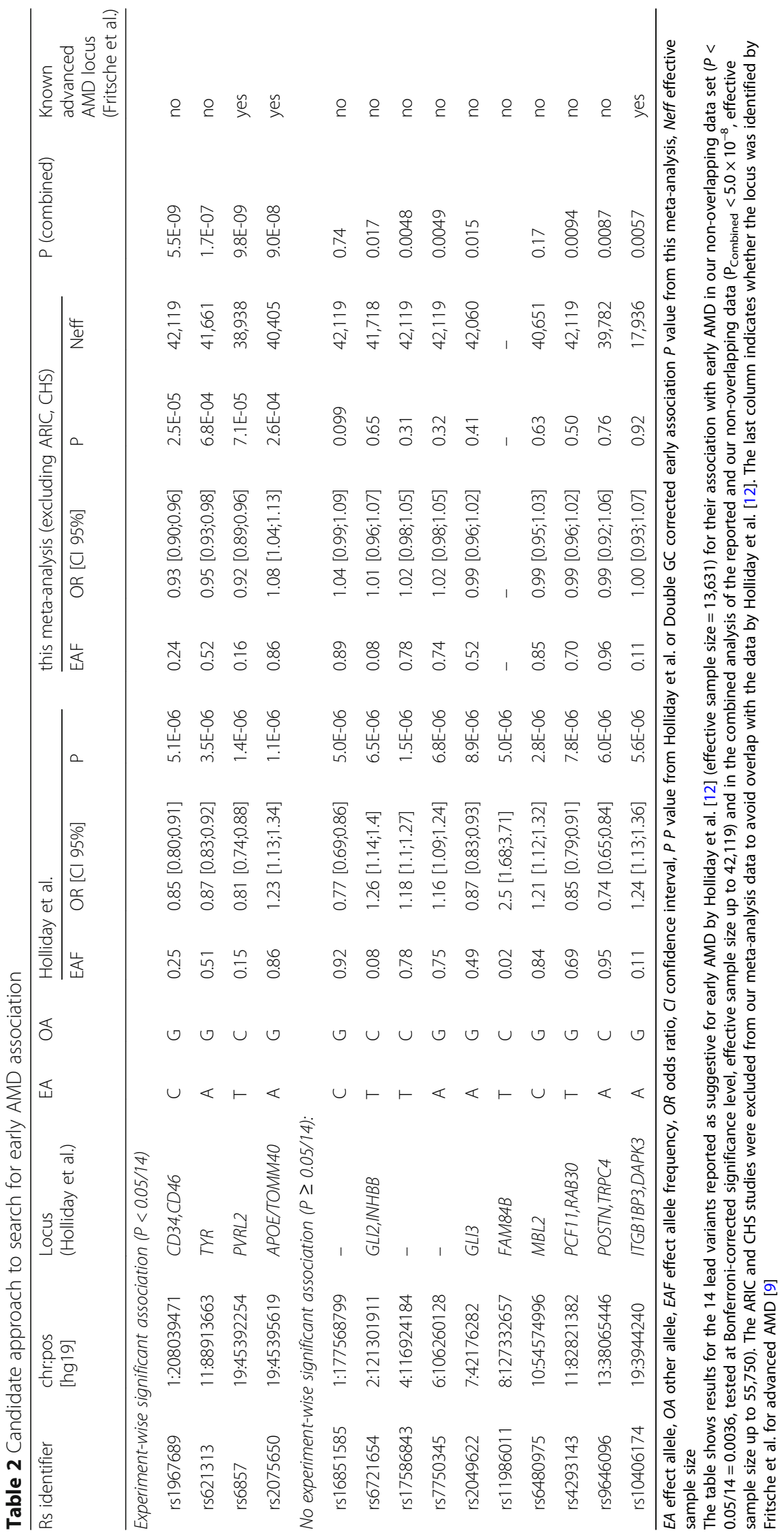


the first time for any AMD with statistical significance (rs621313, near TYR, $P=6.8 \times 10^{-4}$, Figure S2).

Together with our GWAS approach, we identified 10 independent loci with statistical significance for early AMD: (i) eight from our GWAS $\left(P<5.0 \times 10^{-8}\right.$ in our meta-analysis, tier 1), (ii) one additional from the candidate approach with experiment-wise and with genomewide significance (tier 2), and (iii) one additional from the candidate approach with experiment-wise significance (tier 3). Among the 10 identified loci (any tier), two were reported previously for early AMD (near CFH, ARMS2/ HTRA1) and eight were identified here for the first time for early AMD with statistical significance (near $C D 46$, C2, C3, CETP, TNFRSF1OA, VEGFA, APOE/TOMM40/ $P V R L 2$ and TYR). The eight loci included two that have not previously been identified with statistical significance for any AMD (near CD46 and TYR). These two loci showed no second signals (GCTA [31], $\mathrm{P}_{\text {Cond }}>5.0 \times 10^{-8}$ for CD46 and $\mathrm{P}_{\text {Cond }}>0.05 / 14$ for TYR, Figure S1-2).

\section{Sensitivity analysis on the 10 identified early AMD loci}

We conducted various sensitivity analyses to evaluate the robustness of the associations for the 10 identified lead variants: (a) leaving out one of the 11 data sets at a time showed similar effect estimates in most cases, except for the exclusion of the two largest contributing sources, IAMDGC and UKBB, which had the strongest impact on effect sizes due to their relatively large sample size (Figure S3). Exclusion of IAMDGC slightly increased the CD46 effect size (due to a slightly smaller effect in IAMDGC, Figure S3A), had no impact on the TYR effect size (Figure S3B), and decreased effect sizes for C2, CETP, C3, APOE, CFH and ARMS2/HTRA1 variants (due to a relatively large association in IAMDGC). Exclusion of UKBB had no impact on the $C D 46$ variant effect (Figure S3A), slightly diminished the TYR effect (due to a relatively strong effect in UKBB, Figure S3B) and increased effect sizes near $C 2, \mathrm{C} 3$ or $C F H$. (b) Between-study heterogeneity in our meta-analysis was similar to the heterogeneity in any of the leave-one-out meta-analyses, which indicated that the observed heterogeneity was not driven by one single data set (Table S5). We observed low to moderate between-study heterogeneity [28] for the two novel any AMD loci $\left(\mathrm{I}^{2}\right.$ between 22 to $48.3 \%$ and 45.4 to $63.8 \%$ for the TYR and CD46 lead variant, respectively). (c) Effect sizes were robust to additional adjustment for sex or inclusion of additional genetic principal components in the regression analyses (Figure S4). In the following, we were particularly interested in finemapping the two loci that have not been identified before for any AMD: the loci near CD46 and TYR.

\section{Gene prioritization at the two novel loci}

To prioritize variants and genes at the CD46 and TYR locus, we conducted in silico follow-up analyses for all variants and overlapping genes for each of these two loci (4451 or 5729 variants, 10 or 7 genes, respectively). We found several interesting aspects (Table 3): (1) When prioritizing variants according to their statistical evidence for being the driver variant by computing $95 \%$ credible sets of variants [32], we found 23 and 294 credible set variants for the CD46 and TYR locus, respectively (Table S6). (2) Using the Variant Effect Predictor [33], we assessed overlap of credible set variants with functional regulatory regions and found variants influencing the transcript and/or the protein for four genes (Table S7): variants causing an alternative splice form for CD46, a nonsense-mediated mRNA decay (NMD) for CR1L, a missense variant for TYR (rs1042602, $\mathrm{r}^{2}=$ 0.56 to the lead variant rs621313), and NMD variants for NOX4. (3) We investigated credible set variants for being an expression quantitative trait locus (eQTL) for any of the 17 genes in retina (Eye Genotype Expression database, EyeGEx [34]) or in 44 other tissues (Genotype-Tissue Expression database, GTEx [35]). For the CD46 locus, we observed significant association of the lead variant and additional 16 credible set variants on $C D 46$ expression in retina (FDR $<5 \%$, Table S8); the early AMD risk increasing alleles of all 17 variants were associated with elevated CD46 expression. Importantly, we observed the expression signal to colocalize with the early AMD association signal using eCAVIAR [36] (3 variants with colocalization posterior probability CLPP> 0.01 , Table S9, Figure S5-S6). We also found credible variants to be associated with CD46 expression in 15 other tissues from GTEx, including four brain tissues $($ FDR $<0.05$, Table S10). Among the credible set variants in the two loci, we found no further eQTL for any of the other genes. When extending beyond the credible set, we found one further CD46 locus variant as eQTL for CD55, but without colocalization (Table S9, Figure S5S6). These findings support the idea that the credible set captures the essential signal. (4) We queried the 17 genes overlapping the two loci for expression in eye tissue and cells in EyeIntegration summary data [37]. We found five and three genes, respectively, expressed in adult retina and adult RPE cells (CD46, PLXNA2, CR1, CD34, CD55; TYR, GRM5, NOX4; Figure S7-S8). (5) When querying the 17 genes in the Mouse Genome Informatics, MGI [38] or Online Mendelian Inheritance in Man, $\mathrm{OMIM}^{\circ}$, database, for eye phenotypes in mice or humans, we identified relevant eye phenotypes for five genes in mice (CD46, CR1, CR1L, PLXNA2; TYR; Table S11) and for one gene in human (TYR; Table S12).

While it is debatable how to prioritize evidence for a gene's probability to be causal, one approach is to count any of the following characteristics for each of the 17 genes (Gene Prioritization Score, GPS Table 3): any credible set variant is (i) protein-coding, (ii) involved in 
Table 3 Summary of in silico follow-up and gene prioritization score (GPS)

\begin{tabular}{|c|c|c|c|c|c|c|c|c|c|c|c|c|c|}
\hline \multirow[b]{2}{*}{ Locus } & \multirow[b]{2}{*}{$\begin{array}{l}\text { Candidate } \\
\text { gene }\end{array}$} & \multirow[b]{2}{*}{ Chr } & \multirow[b]{2}{*}{ Pos-Start } & \multirow[b]{2}{*}{ Pos-End } & \multirow[b]{2}{*}{$\begin{array}{l}\text { Number } \\
\text { of variants } \\
\text { in } 95 \% \\
\text { credible set }\end{array}$} & \multirow[b]{2}{*}{ GPS } & \multicolumn{4}{|c|}{$\begin{array}{l}\text { Annotation for variants in 95\% } \\
\text { credible set }\end{array}$} & \multicolumn{3}{|c|}{ Biology of the gene } \\
\hline & & & & & & & $\begin{array}{l}\text { Protein } \\
\text { Altering }\end{array}$ & NMD & $\begin{array}{l}\text { Altered } \\
\text { splicing }\end{array}$ & $\overline{\mathrm{eQTL}^{\mathrm{a}}}$ & $\begin{array}{l}\text { Expressed in } \\
\text { Eye tissue } \S\end{array}$ & $\begin{array}{l}\text { MGl Mouse } \\
\text { eye } \\
\text { phenotype }\end{array}$ & $\begin{array}{l}\text { OMIM Human } \\
\text { eye phenotype }\end{array}$ \\
\hline CD46 & CD46 & 1 & $207,925,382$ & $207,968,861$ & 11 & 4 & 0 & 0 & 1 & 1 & 1 & 1 & 0 \\
\hline CD46 & CRIL & 1 & $207,818,457$ & $207,897,036$ & 1 & 1 & 0 & 0 & 0 & 0 & 0 & 1 & 0 \\
\hline CD46 & PLXNA2 & 1 & $208,195,587$ & $208,417,665$ & 0 & 2 & 0 & 0 & 0 & 0 & 1 & 1 & 0 \\
\hline CD46 & $C R 1$ & 1 & $207,669,472$ & $207,815,110$ & 0 & 2 & 0 & 0 & 0 & 0 & 1 & 1 & 0 \\
\hline CD46 & LOC148696 & 1 & $207,991,723$ & $207,995,941$ & 1 & 0 & 0 & 0 & 0 & NA & NA & 0 & 0 \\
\hline CD46 & CD34 & 1 & $208,059,882$ & $208,084,683$ & 0 & 1 & 0 & 0 & 0 & 0 & 1 & 0 & 0 \\
\hline CD46 & CD55 & 1 & $207,494,816$ & $207,534,311$ & 0 & 1 & 0 & 0 & 0 & 0 & 1 & 0 & 0 \\
\hline CD46 & $C R 2$ & 1 & $207,627,644$ & $207,663,240$ & 0 & 0 & 0 & 0 & 0 & 0 & 0 & 0 & 0 \\
\hline CD46 & MIR29B2 & 1 & $207,975,787$ & $207,975,868$ & 0 & 0 & 0 & 0 & 0 & NA & NA & 0 & 0 \\
\hline CD46 & MIR29C & 1 & $207,975,196$ & $207,975,284$ & 0 & 0 & 0 & 0 & 0 & NA & NA & 0 & 0 \\
\hline TYR & $T Y R$ & 11 & $88,911,039$ & $89,028,927$ & 39 & 4 & 1 & 0 & 0 & 0 & 1 & 1 & 1 \\
\hline TYR & NOX4 & 11 & $89,057,521$ & $89,322,779$ & 1 & 3 & 0 & 1 & 1 & 0 & 1 & 0 & 0 \\
\hline TYR & GRM5 & 11 & $88,237,743$ & $88,796,846$ & 109 & 1 & 0 & 0 & 0 & 0 & 1 & 0 & 0 \\
\hline TYR & FOLHIB & 11 & $89,392,464$ & $89,431,886$ & 0 & 0 & 0 & 0 & 0 & 0 & 0 & 0 & 0 \\
\hline TYR & GRM5-AS1 & 11 & $88,237,743$ & $88,257,222$ & 0 & 0 & 0 & 0 & 0 & 0 & NA & 0 & 0 \\
\hline TYR & TRIM49 & 11 & $89,530,822$ & $89,541,743$ & 0 & 0 & 0 & 0 & 0 & 0 & NA & 0 & 0 \\
\hline TYR & TRIM77 & 11 & $89,443,466$ & $89,451,040$ & 0 & 0 & 0 & 0 & 0 & 0 & NA & 0 & 0 \\
\hline
\end{tabular}

${ }^{a}$ Variants in 95\% credible set are a local expression quantitative trait locus for this gene in retina (EyeGEx) or any tissue included in the GTEx database (cis for genes in locus); § Expression in Eye Integration data; NMD nonsense-mediated mRNA decay; OMIM Online Mendelian Inheritance in Man (https://www.omim.org/); NA data not available; The gene start and end positions were extracted from the hg19 gene range list from http://www.cog-genomics.org/plink/1.9/resources The table summarizes statistical and functional evidence for 10 and seven candidate genes of the novel early AMD loci on chromosome 1 and chromosome 11 , respectively. Detailed results on the individual statistical and functional analyses are shown in Tables S6-S12. For the GPS, the sum of cell entries for "annotation" and "biology" was computed per row

NMD, (iii) affecting splice function, (iv) an eQTL for this gene in retina (EyeGEx) or in any other tissue (GTEx), or/and the gene (v) is expressed in retina or RPE, (vi) linked to eye phenotype in mouse or (vii) human. This approach offered CD46 and TYR as the highest scored gene in the respective locus $(\mathrm{GPS}=4$ for each; Table 3).

\section{Phenome-wide association search for the two novel loci}

Co-association of variants in the two novel loci for early AMD with other traits and diseases may provide insights into shared disease mechanisms. We queried different data sets on numerous phenotypes by a gene-based and by a locus-based view.

For the gene-based view, we focused on 82 traits and evaluated reported genome-wide significant $(P<5.0 \times$ $10^{-8}$ ) lead variants (and proxies, $\mathrm{r}^{2}>0.5$ ) for overlap with any of the 17 gene regions (Table S13). For the $C D 46$ locus, we found significant association corrected for multiple testing (false-discovery rate, $\mathrm{FDR}<5 \%$ ) for schizophrenia (in CD46 and CR1L) and for Alzheimer's disease (in CR1, Table S14). For the TYR locus, we found significant associations for eye color, skin pigmentation and skin cancer (in GRM5 and TYR, Tables S14).

For the locus-based view, we conducted a phenomewide association study (PheWAS): we evaluated whether the two lead variants were associated with any of the 778 traits in UK Biobank using GeneAtlas $(n=452,264$, age-adjusted estimates; Table S15) [42]. For the CD46 lead variant, we identified 27 significant trait associations (FDR $<5 \%$ ), including four with particularly strong evidence $\left(P<5.0 \times 10^{-8}\right.$; white blood cell, neutrophil, monocyte count and plateletcrit); the early AMD risk increasing allele $(G$, frequency $=79 \%$ ) was associated consistently with increased blood cell counts. We did not find a significant association of the CD46 lead variant with schizophrenia in UK Biobank (FDR > 5\%; Alzheimer's disease not available). For the $T Y R$ lead variant (rs621313, G allele associated with increased early AMD risk, frequency $=48 \%$ ), we identified 20 significant trait associations including Melanoma (FDR $<5 \%$, G allele associated with increased Melanoma risk) and two with particularly strong evidence for skin color and ease of 
skin tanning $\left(\mathrm{P}<5.0 \times 10^{-8}\right.$, G allele associated with brighter skin color and increased ease of skin tanning).

\section{Advanced AMD association and interaction analyses for the two novel loci}

Next, we investigated whether the early AMD loci CD46 or TYR were associated with advanced AMD. We thus queried the two lead variants for early AMD (rs4844620 and rs621313, respectively) for their advanced AMD association in the IAMDGC data (Table S16). We observed nominally significant directionally consistent effects for advanced $\mathrm{AMD}\left(\mathrm{OR}_{\mathrm{adv}}=1.05,95 \%\right.$ confidence interval, $\mathrm{CI}=[1.01,1.09]$ and $1.03[1.00,1.07], \mathrm{P}_{\mathrm{adv}}=0.02$ and 0.05 , respectively) that were slightly smaller compared to early AMD effects $\left(\mathrm{OR}_{\text {early }}=1.10[1.06,1.14]\right.$ and 1.05 [1.02, $1.08], \mathrm{P}_{\text {early }}=4.7 \times 10^{-8}$ and $\left.6.8 \times 10^{-4}\right)$.

When exploring variant $\mathrm{x}$ age interaction for early AMD (in a subset of our meta-analysis of 10,890 early AMD cases and 54,697 controls) or for advanced AMD (IAMDGC data [44]) for the two novel locus lead variants, we found no statistically significant interaction at a Bonferroni-corrected level for early or advanced AMD $\left(\mathrm{P}_{\text {GxAGE }}>0.05 / 2=0.025\right.$, Table S17-S18).

We were interested in whether one of the two novel lead variants showed interaction with any of the 34 known advanced AMD variants for association with advanced AMD (IAMDGC data). We found no significant interaction $\left(\mathrm{P}_{\mathrm{GxG}}>0.05 / 34 / 2\right.$, Table $\left.\mathrm{S} 19\right)$, which suggests that the known advanced AMD effects are not modulated by the two novel early AMD variants.

\section{Dissecting advanced AMD genetics into shared and distinct genetics for early AMD}

We were interested in whether we could learn about advanced AMD genetics from a joint view of advanced and early AMD genetic effects. First, when computing genetic correlation of advanced AMD genetics with early AMD genetics, we found a substantial correlation of $78.8 \%$ (based on LD-score regression). Second, we contrasted advanced AMD effect sizes (IAMDGC data [9]) with early AMD effect sizes (our meta-analysis,) for the 34 known advanced AMD lead variants (Fig. 2, Table S16). We found two classes of variants: (1) 25 variants showed nominally significant effects on early AMD $(P<0.05$; "advanced-and-early-AMD loci”), all directionally consistent and all smaller for early vs. advanced AMD ( $\mathrm{OR}_{\text {early }}=$ 1.04-1.47; $\mathrm{OR}_{\mathrm{adv}}=1.10-2.81$ ); (2) nine variants had no nominally significant effect on early AMD $(P \geq 0.05$; "advanced-only AMD loci"). We did not find any variant with early AMD effects into the opposite direction as the advanced AMD effects. Also, we did not find any variant-age interaction on early AMD (Table S18).

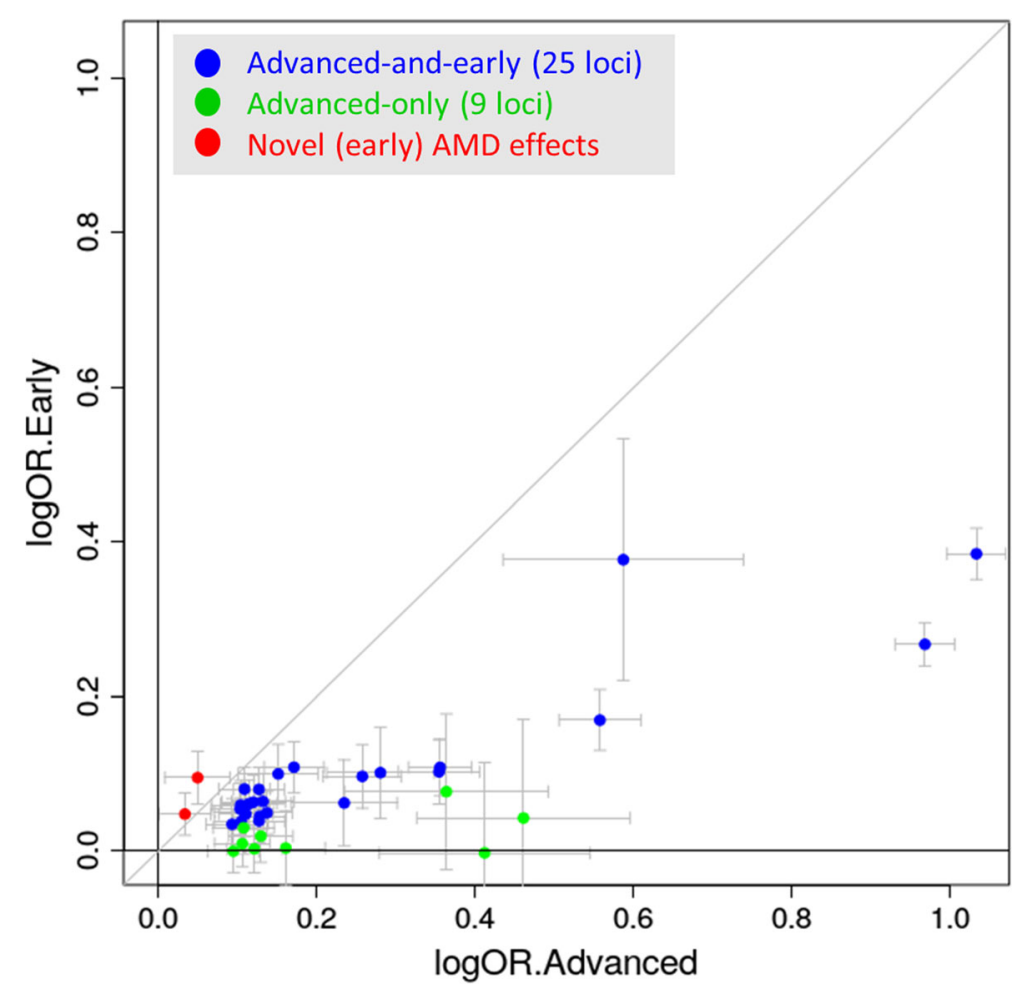

Fig. 2 Advanced vs early AMD effect sizes. Shown are advanced AMD effect sizes contrasted to early AMD effect sizes (effect sizes as log odds ratios) for the 34 known advanced AMD variants [9] (blue or green for $P_{\text {early }}<0.05$ or $P_{\text {early }} \geq 0.05$, respectively) and for the two novel (early) $A M D$ variants (red, near CD46, TYR). Detailed results are shown in Table S16 
We observed that complement genes CFH, CFI, C3, $C$, and $C 2$ were all included in the 25 advanced-andearly-AMD loci. We were thus interested in whether advanced-and-early-AMD loci suggested different pathways compared to advanced-only-AMD loci. For this, we utilized the GPS from our previous work on advanced AMD [9] to select the best-supported genes in each of these loci (Table S20). We applied Reactome pathway analyses via Enrichr [47] twice: (i) for the 35 genes in the 25 advanced-and-early-AMD loci and (ii) for the nine genes in eight advanced-only-AMD loci (no gene in the "narrow" locus definition of the RORB locus). This revealed significant enrichment (corrected $\mathrm{P}<0.05$ ) for genes from "complement system" and "lipoprotein metabolism" in the 25 advanced-and-early-AMD loci and enrichment for genes in the pathways "extracellular matrix organization" and "assembly of collagen fibrils" in the 8 advanced-only-AMD loci (Table 4). This suggested that the early AMD effect of advanced AMD variants distinguished the major known pathways for advanced AMD.

\section{Discussion}

Based on the largest genome-wide meta-analysis for early AMD to date encompassing $\sim 14,000$ cases and 91,000 controls, all color fundus photography confirmed and a candidate approach based on 14 suggestive variants from Holliday et al. [12], we identified 10 loci for early AMD including eight novel and two previously identified for early AMD [12]. Eight of the 10 identified loci overlapped with known loci for advanced AMD [9] and two had not been detected by GWAS for early or advanced AMD so far. Our post-GWAS approach highlighted CD46 and TYR as compelling candidate genes in the two loci. Our joint view on early and advanced AMD genetics allowed us to differentiate between shared and distinct genetics for these two disease stages, which the pathway analyses suggested to be biologically relevant.

We defined three tiers of identified variants that reflected the different levels of data and evidence: tier 1 variants were identified with genome-wide significance in our GWAS meta-analysis data (identifying 8 loci including CD46), tier 2 variants were among the 14 candidate-based variants judged at experiment-wise significance in our non-overlapping data $(P<0.05 / 14=$ $0.0036)$ as well as at genome-wide significance in the combined analysis (i.e. previously published summary statistics and ours; identifying one additional locus near $A P O E$ ), and tier 3 variants were among the 14 variants judged at experiment-wise significance, but no genomewide significance in the combined data (identifying one additional locus near TYR). While the establishing of genome-wide significance for a locus is ideal, testing at a statistical significance level controlling the experimentwise type 1 error with Bonferroni-correction is also an established approach to provide statistical evidence in independent data [48].

Particularly interesting were the two loci near $C D 46$ and TYR that were identified here for early AMD with statistical significance and have not been identified previously, not even for advanced AMD. The locus around CD46 had been reported as suggestive for early AMD by the previous largest GWAS for early AMD [12] (4089 early AMD cases, 20,453 controls), but not with statistical significance, and had not been identified with statistical significance by the previous largest GWAS for advanced AMD [9] (16,144 advanced AMD cases, 17,832

Table 4 Enriched pathways

\begin{tabular}{|c|c|c|c|c|c|c|}
\hline Gene group & Reactome pathway & $\begin{array}{l}\text { \#Genes in } \\
\text { gene set }\end{array}$ & $\begin{array}{l}\text { \#AMD loci } \\
\text { in gene set }\end{array}$ & Raw P & Corrected P & $\begin{array}{l}\text { Genes contributing to } \\
\text { enrichment }\end{array}$ \\
\hline \multirow[t]{6}{*}{$\begin{array}{l}\text { Effects on early and } \\
\text { advanced AMD }\end{array}$} & $\begin{array}{l}\text { Regulation of Complement cascade } \\
\text { (R-HSA-977606) }\end{array}$ & 26 & 5 & $7.8 \times 10^{-10}$ & $1.2 \times 10^{-6}$ & C3;CFH;C9;CFI;CFB \\
\hline & Lipoprotein metabolism (R-HSA-174824) & 34 & 4 & $3.5 \times 10^{-7}$ & $1.8 \times 10^{-4}$ & $A B C A 1 ; C E T P ; L I P C ; A P O E$ \\
\hline & Complement cascade (R-HSA-166658) & 80 & 5 & $2.7 \times 10^{-7}$ & $2.0 \times 10^{-4}$ & $\mathrm{C3} ; \mathrm{CFH} ; \mathrm{CP} ; \mathrm{CFI} ; \mathrm{CFB}$ \\
\hline & HDL-mediated lipid transport (R-HSA-194223) & 19 & 3 & $4.7 \times 10^{-6}$ & $1.8 \times 10^{-3}$ & $A B C A 1 ; C E T P ; A P O E$ \\
\hline & $\begin{array}{l}\text { Lipid digestion, mobilization, and transport } \\
\text { (R-HSA-73923) }\end{array}$ & 71 & 4 & $7.0 \times 10^{-6}$ & $2.2 \times 10^{-3}$ & ABCA1;CETP;LIPC;APOE \\
\hline & Activation of C3 and C5 (R-HSA-174577) & 6 & 2 & $4.4 \times 10^{-5}$ & 0.011 & $C 3 ; C F B$ \\
\hline \multirow[t]{3}{*}{$\begin{array}{l}\text { no effects on early } \\
\text { AMD }\end{array}$} & $\begin{array}{l}\text { Assembly of collagen fibrils and other } \\
\text { multimeric structures (R-HSA-2022090) }\end{array}$ & 54 & 3 & $1.5 \times 10^{-6}$ & $2.4 \times 10^{-3}$ & COL15A1;COL8A1;MMP9 \\
\hline & Collagen formation (R-HSA-1474290) & 85 & 3 & $6.1 \times 10^{-6}$ & $3.1 \times 10^{-3}$ & COL15A1;COL8A1;MMP9 \\
\hline & $\begin{array}{l}\text { Extracellular matrix organization } \\
\text { (R-HSA-1474244) }\end{array}$ & 283 & 4 & $4.7 \times 10^{-6}$ & $3.6 \times 10^{-3}$ & VTN;COL15A1;COL8A1;MMP9 \\
\hline
\end{tabular}

The table shows enriched pathways for highest prioritized genes (from Fritsche et al. 2016 without modifications) in the 25 late AMD loci with early AMD effects (35 genes) versus the 8 loci with no effect on early AMD (9 genes). Pathways with significant corrected $P$-value $\left(P_{\text {corr }}<0.05\right)$ for each gene group from EnrichR querying human Reactome database 2016 are shown 
controls). Our meta-analysis was more than three times larger than the previous early AMD GWAS (effective sample size 48,651 compared to 13,631 [12]) and had a larger power to detect an "any AMD" effect with genome-wide significance than the previous advanced AMD GWAS (e.g. for OR $=1.10$, allele frequency $30 \%$ : power $=92 \%$ compared to $61 \%$, respectively). The $T Y R$ locus had not been identified with statistical significance in any previous GWAS on early or advanced AMD; it was stated as a locus with suggestive evidence from the previous GWAS on early AMD $\left(P=3.5 \times 10^{-6}\right)[12]$ and was identified here with statistical significance at experiment-wise error control. The combined analysis of the Holliday et al. and our non-overlapping data clearly separated the 14 Holliday variants into four with genome-wide or close to genome-wide significance and 10 that were far away from statistical significance.

Prioritization of genes underneath association signals is a known challenge, but highly relevant for selecting promising candidates for functional follow-up. Our systematic approach, scrutinizing all genes underneath our two newly identified loci, highlighted CD46 and TYR as the most supported genes. CD46 is an immediate compelling candidate as a part of the complement system [49]. Complement activation in retina is thought to have a causal role for AMD [50, 51]. Importantly, we found our CD46 GWAS signal to colocalize with CD46 expression with the early AMD risk increasing allele (rs4844620 G) increasing CD46 expression in retinal cells. On the one hand, this contrasts the presumption that a higher CD46 expression in eye tissue should protect from AMD, based on previous CD46 expression data [52] and a documented AMD risk increasing effect for increased complement inhibition [53]. On the other hand, CD46 had also been found to have pathogenic receptor properties for human viral and bacterial pathogens (e.g. measles virus) [54] and is known to downmodulate adaptive $\mathrm{T}$ helper type 1 cells [55]. Furthermore, a GWAS on neutralizing antibody response to measles vaccine had identified two intronic $C D 46$ variants (rs2724384, rs2724374) [56]. In our data, these two variants were in the $95 \%$ credible set for the $C D 46$ locus, highly correlated with our lead variant $\mathrm{rs} 4844620\left(\mathrm{r}^{2}>=\right.$ 0.95), and the major alleles (rs2724374 T, rs2724384 A) increased early AMD risk. Interestingly, the rs2724374 G was shown for $C D 46$ exon skipping resulting in a shorter CD46 isoform with a potential role in pathogen binding [56]. Based on this, one may hypothesize that the observed CD46 signal in early AMD is related to pathogenic receptor properties rather than complement inactivation.

At the second locus, TYR appears as the best supported gene by our systematic scoring. This locus and gene was already discussed by Holliday and colleagues [12]. Briefly,
TYR is important for melanin production and TYR variants in human were associated with skin, eye and hair color [57-59]. While we did not identify any cis effect between the credible set variants and TYR expression, one of our credible set variants in TYR (rs1042602) is a missense variant. Interestingly, this variant was a GWAS lead variant not only for skin color [58], but also for macular thickness in UK Biobank [60]; the allele associated with thicker retina showed increased early AMD risk in our data. Since thicker RPE/Bruch's membrane complex was associated with increased early AMD risk in the AugUR study [61], this would be in line with our early AMD risk increasing allele being linked to a process of increased accumulation of drusenoid debris in the RPE/Bruch's membrane complex. Although CD46 and TYR were the most supported genes in the two loci, we could not rule-out the relevance of other genes in the loci.

It is a strength of the current study that early AMD and control status was ascertained by color fundus photography, not relying on health record data. However, the early AMD classification in our GWAS was heterogeneous across the 11 data sets: one study incorporated information from optical coherence tomography (NICOLA), the UK Biobank classification was derived by a machine-learning algorithm [20], and the IAMDGC data was multi-site with different classification approaches [9]. The uncertainty in early AMD classification and the substantial effort required for any manual AMD classification are likely reasons for the sparsity of early AMD GWAS so far. Our sensitivity analysis with the leaveone-out meta-analyses and corresponding heterogeneity estimates showed that effect estimates did not depend on one or the other data source or classification approach.

Our data on early AMD genetics is comparable in size to the existing data on advanced AMD genetics from IAMDGC (summary statistics at http://amdgenetics.org/ ) and thus provides an important resource (summary statistics at http://genepi-regensburg.de) to enable a joint view. By this joint view, we were able to differentiate the 34 loci known for advanced AMD into 25 "advancedand-early-AMD loci" and nine "advanced-AMD-only loci". Pathway enrichment analyses conducted separately for these two groups effectively discriminated the major known pathways for advanced AMD genetics [9]: complement complex and lipid metabolism for "advancedand-early-AMD" loci; extracellular matrix metabolism for "advanced-AMD-only" loci. The two novel loci around CD46 and TYR fit to the definition of "advanced-and-early-AMD" loci and the CD46 being part of the complement system supports the above stated pathway pattern. The larger effect size for early compared to advanced AMD for the two novel loci may - in part be winner's curse. 
How do our observations relate to potential etiological models? (1) For a genetic variant capturing an underlying mechanism that triggers both early and advanced AMD, we would expect the variant to show association with early and advanced AMD (compared to "healthy") with directionally consistent effects (Fig. 3, Model 1). This would be in line with our observed associations for the 27 "advanced-and-early-AMD" loci (25 known advanced AMD loci, 2 novel loci). This would also suggest that mechanisms of complement system or lipid metabolism trigger both early and advanced disease. (2) For a mechanism that triggers advanced AMD no matter whether the person is "healthy" or has early AMD, we would anticipate a variant effect for advanced AMD, but not for early AMD (Fig. 3, Model 2). This would be in line with our observed associations for the nine "advanced-AMD-only" loci. This would also suggest that mechanisms of extracellular matrix metabolism trigger advanced AMD rather than early AMD. Of note, these include the MMP9 locus, which is thought to trigger vascularization and wet AMD [9]. (3) Another mechanism is conferred by variants that are purely responsible for progression from early to advanced AMD, but do not increase advanced AMD risk for "healthy" individuals. In such a scenario, the advanced AMD risk increasing allele would be under-represented among persons with early AMD (Fig. 3, Model 3), particularly at older age, and it would be associated with decreased risk of early AMD (compared to "healthy"). None of the identified variants showed this pattern overall or for older age in the variant $x$ age interaction analyses. (4) For a mechanism that triggers early AMD, but has no impact on the progression from early to advanced AMD, we would have an effect on early AMD, but no effect on advanced AMD (Fig. 3, Model 4). We did not find such a variant.

Our data and joint view on effects for both disease stages support two of the four etiological models. One may hypothesize that the unsupported models are nonexisting or unlikely. There are limitations to consider: (1) To reduce complexity, we adopted an isolated view per variant with some accounting for interaction, but ignoring more complex networks. (2) Early AMD effects were estimated predominantly in population-based studies, while advanced AMD effects were from a casecontrol design. (3) The cut-off of "nominal significance" for separating variants into "advanced-and-early" or "advanced-only" loci is arbitrary and larger data might give rise to re-classification. Still, the power to detect effects for early AMD in our meta-analysis was similar to the power in the advanced AMD data from IAMDGC (for $\mathrm{OR}=1.05$, allele frequency $30 \%$, nominal significance: power $=94 \%$ or $83 \%$, respectively). (4) An improved disentangling of genetic effects for the two chronologically linked disease stages will be an important subject of further research, requiring large-scale population-based studies with long-term follow-up and the estimation of transition probabilities.

\section{Conclusions}

In summary, our large GWAS on early AMD identified novel loci, highlighted shared and distinct genetics between early and advanced AMD and provides insights into AMD etiology. The ability of early AMD effects to differentiate the major pathways for advanced AMD underscores the biological relevance of a joint view on early and advanced AMD genetics.

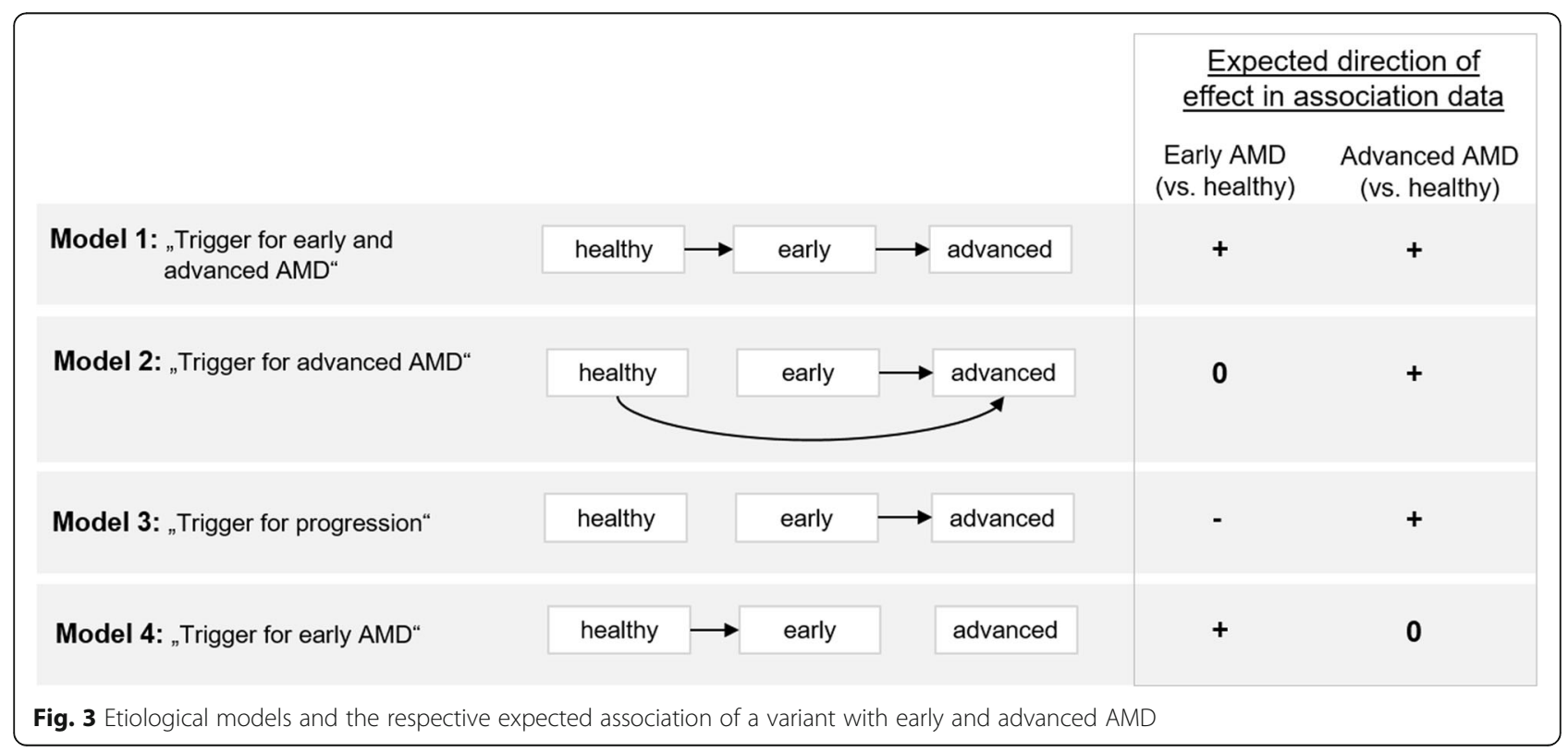




\section{Supplementary information}

Supplementary information accompanies this paper at https://doi.org/10. 1186/s12920-020-00760-7.

\section{Additional file 1: Supplementary Tables. Additional file 2: Supplementary Note. Additional file 3: Supplementary Figures.}

\section{Abbreviations}

3CC: Three Continent Consortium; AMD: Age-related macular degeneration; ARIC: The Atherosclerosis Risk in Communities Study; AugUR: Age-related diseases: Understanding Genetic and non-genetic influences - a study at the University of Regensburg; CHS: Cardiovascular Health Study; eQTL: Expression quantitative trait locus; FDR: False-discovery-rate; GCTA: Genome-wide Complex Trait Analysis; GC: Genomic control; GHS: Gutenberg Health Study; GPS: Gene Prioritization Score; GWAS: Genome-wide association study; HRC: Haplotype Reference Consortium; IAMDGC: International AMD Genomics Consortium; KORA: KOoperative Gesundheitsforschung in der Region Augsburg; LIFE: Leipzig Research Centre for Civilization Based Diseases - LIFE Adult population-based study, city of Leipzig, Germany; LOO: Leave-one-out: MAC: Minor allele count; MGl: Mouse Genome Informatics; NICOLA: Northern Ireland Cohort for Longitudinal Study of Ageing; NMD: Nonsense-mediated mRNA decay; OCT: Optical coherence tomography; OMIM: Online Mendelian Inheritance in Man; QC: Quality control; RPE: Retinal pigment epithelium; UKBB: UK Biobank; VEP: Variant effect predictor; WHI: Women's Health Initiative

\section{Acknowledgments}

The authors thank the staff and participants of the ARIC, AugUR, CHS, GHS, IAMD GC, KORA S4, LIFE-Adult NICOLA, UKBB and WHI studies for their important contributions. We are grateful to all the participants of the NICOLA Study, and the whole NICOLA team, which includes nursing staff, research scientists, clerical staff, computer and laboratory technicians, managers and receptionists. The authors alone are responsible for the interpretation of the data and any views or opinions presented are solely those of the authors and do not necessarily represent those of the NICOLA Study team. The authors wish to express their sincere thanks to the participants of LIFE-Adult for their time and blood samples. The authors gratefully acknowledge Dr. Kerstin Wirkner and her team at the Leipzig Research Center for Civilization Diseases (LIFE-Adult), Leipzig University, Leipzig, Germany for data acquisition. A part of LIFE-Adult genotyping was done at the Cologne Center for Genomics (CCG, University of Cologne, Prof. Dr. Peter Nürnberg and Dr. Mohammad R. Toliat). For LIFE-Adult genotype imputation, computation infrastructure provided by ScaDS (Dresden/Leipzig Competence Center for Scalable Data Services and Solutions) at the Leipzig University Computing Centre was used. This study has been conducted using the UK Biobank resource under Application Number 33999. The UK Biobank was established by the Wellcome Trust medical charity, Medical Research Council, Department of Health, Scottish Government and the Northwest Regional Development Agency. It has also had funding from the Welsh Assembly Government, British Heart Foundation and Diabetes UK. The Atherosclerosis Risk in Communities study has been funded in whole or in part with Federal funds from the National Heart, Lung, and Blood Institute, National Institute of Health, Department of Health and Human Services, under contract numbers (HHSN2682017000011, HHSN2682017000021, HHSN268201700003I, HHSN268201700004I, and HHSN268201700005I). Funding for CARe genotyping was provided by NHLBI Contract N01-HC-65226. Funding for GENEVA was provided by National Human Genome Research Institute grant U01HG004402 (E. Boerwinkle). This study is part of the PAGE program, a cooperative agreement funded by the National Human Genome Research Institute. The PAGE collaborative study is supported by U01HG004803 (G. Heiss), U01HG004798 (D. Crawford), U01HG004802 (L. Le Marchand), U01HG004790 (C. Kooperberg and U. Peters), and U01HG004801 (T. Matise), with additional support from the National Institute of Mental Health. This study is part of the Building on GWAS: the U.S. CHARGE consortiumSequencing (CHARGE-S). Funding for CHARGE-S was provided by NHLBI grant 5RC2HL102419 through the American Recovery and Reinvestment Act of 2009 (ARRA). Additional funding was provided for this study as part of Disease 2020: Large-Scale Sequencing and Analysis Center Initiated Projects; sequencing was completed at the Human Genome Sequencing Center at Baylor College of Medicine under NHGRI grant U54HG003273 and UM1HG008898.Data for the Building on GWAS: the U.S. CHARGE consortium -Sequencing was provided by Eric Boerwinkle on behalf of the Atherosclerosis Risk in Communities (ARIC) Study, L. Adrienne Cupples, principal investigator for the Framingham Heart Study, and Bruce Psaty, principal investigator for the Cardiovascular Health Study. A portion of this research was conducted using the Linux Cluster for Genetic Analysis (LinGA-II) funded by the Robert Dawson Evans Endowment of the Department of Medicine at Boston University School of Medicine and Boston Medical Center. This research was supported by contracts HHSN268201200036C, HHSN268200800007C, N01-HC85079, N01-HC-85080, N01-HC-85081, N01-HC-85082, N01-HC-85083, N01-HC-85084, N01-HC85085, N01-HC-85086, N01-HC-35129, N01 HC-15103, N01 HC-55222, N01HC-75150, N01-HC-45133, and N01-HC-85239; grant numbers U01 HL080295 and U01 HL130014 from the National Heart, Lung, and Blood Institute, and R01 AG-023629 from the National Institute on Aging, with additional contribution from the National Institute of Neurological Disorders and Stroke. A full list of principal CHS investigators and institutions can be found at http://www.chs-nhlbi.org/pi.htm. The WHI program is funded by the National Heart, Lung, and Blood Institute, National Institutes of Health, U.S. Department of Health and Human Services through contracts HHSN268201600018C, HHSN268201600001C, HHSN268201600002C, HHSN268201600003C, and HHSN268201600004C. Funding support for WHI GARNET was provided through the NHGRI Genomics and Randomized Trials Network (GARNET) (Grant Number U01HG005152). Assistance with phenotype harmonization and genotype cleaning, as well as with general study coordination, was provided by the GARNET Coordinating Center (U01HG005157). Assistance with data cleaning was provided by the National Center for Biotechnology Information. Funding support for genotyping, which was performed at the Broad Institute of MIT and Harvard, was provided by the NIH Genes, Environment and Health Initiative [GEI] (U01HG004424). The Women's Health Initiative Sequencing Project (WHISP) was funded by Grant Number RC2 HL102924. This study was part of the NHLBI Grand Opportunity Exome Sequencing Project (GO-ESP). Funding for GO-ESP was provided by NHLBI grants RC2 HL103010 (HeartGO), RC2 HL102923 (LungGO) and RC2 HL102924 (WHISP). The exome sequencing was performed through NHLBI grants RC2 HL102925 (BroadGO) and RC2 HL102926 (SeattleGO). Funding for WHI SHARe genotyping was provided by NHLBI Contract N02-HL-64278. The WHI Sight Exam and the Memory Study was funded in part by Wyeth Pharmaceuticals, Inc., St. Davids, PA. This manuscript was not prepared in collaboration with ARIC, CHS or WHI investigators and does not necessarily reflect the opinions or views of CHS or the NHLBI.

\section{Authors' contributions}

TWW, FG1, CB, CK, FG2, IMH, KJS and BHFW designed the study and wrote the manuscript. TWW and FG1 conducted the meta-analysis. FG2 applied the automated grading of UK Biobank fundus images. TWW and CK conducted the PheWAS. TWW, LW, MEZ and KJS conducted analysis for the gene priority scoring. KJS conducted the pathway analyses. FG1, TS and TWW analysed data from the dbGAP studies ARIC, CHS and WHI. FG1 and TWW analysed data from IAMDGC, AugUR and KORA. TWW analyses data from UKBB. CAK, AP and AKS analysed data from the GHS study. MMN and AP analysed data from the KORA study. FGR, TE, KH, and MS analysed data from the LIFE-Adult study. MCG, AJMK, NQ and REH analysed all data within the NICOLA study for this project. FG2, HK, KJS, IMH and TWW wrote the discussion of etiological models. IMH, KJS and BHFW supervised the study. All authors approved the final version of the manuscript.

\section{Funding}

The AugUR study was supported by grants from the German Federal Ministry of Education and Research (BMBF 01ER1206, BMBF $01 E R 1507$ to I.M.H.) and the University of Regensburg. The Gutenberg Health Study is funded through the government of Rhineland-Palatinate (,Stiftung Rheinland-Pfalz für Innovation", contract AZ 961-386261/733), the research programs "Wissenschafft Zukunft" and "Center for Translational Vascular Biology (CTVB)" of the Johannes Gutenberg-University of Mainz, and its contract with Boehringer Ingelheim and PHILIPS Medical Systems, including an unrestricted grant for the Gutenberg Health Study. Alexander K Schuster (A.K.S.) holds the professorship for ophthalmic healthcare research endowed by "Stiftung Auge "and financed by "Deutsche Ophthalmologische Gesellschaft "and "Berufsverband der Augenärzte Deutschland e.V.". The International AMD Genomics Consortium (IAMDGC) is supported by a grant from NIH (R01 
EY022310). Genotyping was supported by a contract (HHSN268201200008l) to the Center for Inherited Disease Research (http://amdgenetics.org/). In-depth analyses to estimate genetic effects in the IAMDGC data was supported by DFG HE 3690/ 5-1 to Iris M Heid (I.M.H.). The KORA study was initiated and financed by the Helmholtz Zentrum München - German Research Center for Environmental Health, which is funded by the German Federal Ministry of Education and Research (BMBF) and by the State of Bavaria. Furthermore, KORA research was supported within the Munich Center of Health Sciences (MC-Health), LudwigMaximilians-Universität, as part of LMUinnovativ. This publication is supported by the Leipzig Research Centre for Civilization Diseases (LIFE), an organizational unit affiliated to the Medical Faculty of Leipzig University. LIFE is funded by means of the European Union, by the European Regional Development Fund (ERDF) and by funds of the Free State of Saxony within the framework of the excellence initiative (project numbers: 713-241202, 14505/2470, 14575/2470). Franziska G. Rauscher (F.G.R.) is supported by a grant from the German Federal Ministry of Education and Research: i:DSem - Integrative data semantics in systems medicine (031 L0026). Tobias Elze (T.E.) is funded by the Lions Foundation, GrimshawGudewicz Foundation, Research to Prevent Blindness, BrightFocus Foundation, Alice Adler Fellowship, NEI R21EY030142,NEI R21EY030631, NEI R01EY030575, and NEI Core Grant P30EYE003790. The Atlantic Philanthropies, the Economic and Social Research Council, the UKCRC Centre of Excellence for Public Health Northern Ireland, the Centre for Ageing Research and Development in Ireland, the Office of the First Minister and Deputy First Minister, the Health and Social Care Research and Development Division of the Public Health Agency, the Wellcome Trust/Wolfson Foundation and Queen's University Belfast provide core financial support for NICOLA. Marisa Cañadas-Garre (M.C.G.) is supported by Science Foundation Ireland and the Department for the Economy, Northern Ireland US partnership award (15/IA/3152). The molecular data employed for the NICOLAcohort were funded by Economic and Social Research Council (ES/L008459/1). The analyses were supported by German Research Foundation (DFG HE-3690/5-1 to I.M.H.) and by the National Institutes of Health (NIH R01 EY RES 511967 to I.M.H.). Felix Grassmann (F.G.) was a Leopoldina Postdoctoral Fellow (Grant No. LPDS 2018-06) funded by the Academy of Sciences Leopoldina. The position of Tobias Strunz (T.S.) is financed by the Helmut-Ecker-Foundation (\# 05/17 to B.H.F.W.) and of Christina Kiel (C.K.) by a grant from the German Research Foundation to F.G. and B.H.F.W. (GR $5065 / 1-1)$. The funding bodies played no role in the design of the study and collection, analysis, and interpretation of data and in writing the manuscript. Open access funding provided by Projekt DEAL.

\section{Availability of data and materials}

The genome-wide meta-analysis summary statistics for early AMD are available for download from www.genepi-regensburg.de/earlyamd. We included three publicly available studies from dbGaP into the meta-analysis: ARIC (accession number: phs000090.v5.p1), CHS (accession number: phs000287.v6.p1), WHI (accession number: phs000746.v2.p3 and phs000200.v11.p3). Data from other studies included in the meta-analysis can be requested from the respective study centers: AugUR (https://www.uni-regensburg.de/medizin/epidemiologie-praeventivmedizin/genetische-epidemiologie/augur), GHS (http://www.gutenberg-gesundheitsstudie.de/), IAMD GC (http://amdgenetics.org/), KORA (https://www.helmholtz-muenchen.de/ kora), LIFE-Adult (https://life.uni-leipzig.de/de/erwachsenenkohorten/life_ adult.html), NICOLA (https://www.qub.ac.uk/sites/NICOLA/) or UKBB (https:// www.ukbiobank.ac.uk/).

\section{Ethics approval and consent to participate}

The Institutional Review Board (IRB) of the University of Utah was the umbrella IRB for all other studies contributing data to the International Agerelated Macular Degeneration Genomics Consortium (IAMDGC), except for the Beaver Dam Eye Study (BDES). The University of Utah approved and certified each individual study ethic committee's conduct for the data used in this study. Data provided by BDES was approved by the IRB of the University of Wisconsin. Local ethics approval for data access to the studies deposited in dbGAP (WHI, ARIC and CHS) was granted by the IRB of the University of Regensburg. For all other studies, study participants obtained informed consent and local ethics committees approved the study protocols.

Consent for publication

Not applicable.

\section{Competing interests}

M.S. receives funding from Pfizer Inc. for a project not related to this research. Retinal grading of the NICOLA study was supported by Novartis (for R.E.H) and Bayer (for Usha Chakravarthy, not a co-author). A.K.S. received financial and research support by Heidelberg Engineering, Novartis, Bayer Vital and Allergen without a link to the content of this work. I.M.H. received funding from Roche Diagnostics for a project not related to this research. None of the other authors have any conflicts of interest.

\section{Author details}

${ }^{1}$ Department of Genetic Epidemiology, University of Regensburg, Regensburg, Germany. ${ }^{2}$ Institute of Human Genetics, University of Regensburg, Regensburg, Germany. ${ }^{3}$ Department of Medical Epidemiology and Biostatistics, Karolinska Institutet, Stockholm, Sweden. ${ }^{4}$ Institute of Medical Sciences, University of Aberdeen, Aberdeen, Scotland, UK. ${ }^{5}$ Department of Ophthalmology, University Hospital Regensburg, Regensburg, Germany. 'Statistical Consulting Unit StaBLab, Department of Statistics, Ludwig-Maximilians-Universität Munich, Munich, Germany. ${ }^{7}$ Department of Ophthalmology, University Medical Center of the Johannes Gutenberg-University Mainz, Mainz, Germany. ${ }^{8}$ Institute for Medical Biostatistics, Epidemiology and Informatics, University Medical Center of the Johannes Gutenberg-University Mainz, Mainz, Germany. ${ }^{9}$ Institute of Genetic Epidemiology, Helmholtz Zentrum München, German Research Center for Environmental Health, Neuherberg, Germany. ${ }^{10}$ Department of Internal Medicine I (Cardiology), Hospital of the Ludwig-Maximilians-University (LMU) Munich, Munich, Germany. ${ }^{11}$ Genetic Epidemiology, IBE, Faculty of Medicine, LMU Munich, Munich, Germany. ${ }^{12}$ German Center for Diabetes Research (DZD), Neuherberg, Germany. ${ }^{13}$ Institute of Epidemiology, Helmholtz Zentrum München Research Center for Environmental Health, Neuherberg, Germany. ${ }^{14}$ Leipzig Research Centre for Civilization Diseases (LIFE), Leipzig University, Leipzig, Germany. ${ }^{15}$ Institute for Medical Informatics, Statistics, and Epidemiology (IMISE), Leipzig University, Leipzig, Germany. ${ }^{16}$ Schepens Eye Research Institute, Harvard Medical School, Boston, MA, USA. ${ }^{17}$ Centre for Public Health, Queen's University of Belfast, Belfast, UK. ${ }^{18}$ Institute of Clinical Human Genetics, University Hospital Regensburg, Regensburg, Germany.

\section{Received: 16 October 2019 Accepted: 4 August 2020}

Published online: 26 August 2020

\section{References}

1. Lim LS, Mitchell P, Seddon JM, Holz FG, Wong TY. Age-related macular degeneration. Lancet Elsevier. 2012;379:1728-38.

2. Garrity ST, Sarraf D, Freund KB, Sadda SR. Multimodal imaging of Nonneovascular age-related macular degeneration. Invest Ophthalmol Vis Sci. 2018;59:AMD48-64.

3. Forte R, Querques G, Querques L, Massamba N, Le Tien V, Souied EH. Multimodal imaging of dry age-related macular degeneration. Acta Ophthalmol. 2012;90:281-7.

4. Klein R, Meuer SM, Myers CE, Buitendijk GHS, Rochtchina E, Choudhury F, et al. Harmonizing the classification of age-related macular degeneration in the three-continent AMD consortium. Ophthalmic Epidemiol. 2014;21:14-23.

5. Brandl C, Zimmermann ME, Günther F, Barth T, Olden M, Schelter SC, et al. On the impact of different approaches to classify age-related macular degeneration: results from the German AugUR study. Sci Rep. 2018;8:1-10.

6. AREDS. Risk factors associated with age-related macular degeneration. A case-control study in the age-related eye disease study: age-related eye disease study report number 3. Age-Related Eye Disease Study Research Group. Ophthalmology. 2000;107:2224-32.

7. Smith W, Assink J, Klein R, Mitchell P, Klaver CC, Klein BE, et al. Risk factors for age-related macular degeneration: pooled findings from three continents. Ophthalmology Elsevier. 2001;108:697-704.

8. Yonekawa Y, Miller J, Kim I. Age-related macular degeneration: advances in management and diagnosis. J Clin Med. 2015;4:343-59.

9. Fritsche LG, Igl W, Bailey JNC, Grassmann F, Sengupta S, Bragg-Gresham JL, et al. A large genome-wide association study of age-related macular degeneration highlights contributions of rare and common variants. Nat Genet. 2016;48:134-43.

10. Grassmann F, Fritsche LG, Keilhauer CN, Heid IM, Weber BHF. Modelling the genetic risk in age-related macular degeneration. PLoS One. 2012;7.

11. Han X, Gharahkhani P, Mitchell P, Liew G, Hewitt AW, MacGregor S. Genome-wide meta-analysis identifies novel loci associated with age-related macular degeneration. J. Hum. Genet. 2020;10:1. 
12. Holliday EG, Smith AV, Cornes BK, Buitendijk GHS, Jensen RA, Sim X, et al. Insights into the Genetic Architecture of Early Stage Age-Related Macular Degeneration: A Genome-Wide Association Study Meta-Analysis. PLoS One. 2013;8:e53830.

13. Auton A, Abecasis GR, Altshuler DM, Durbin RM, Bentley DR, Chakravarti A, et al. A global reference for human genetic variation. Nature. 2015;526:68-74.

14. McCarthy S, Das S, Kretzschmar W, Delaneau O, Wood AR, Teumer A, et al. A reference panel of 64,976 haplotypes for genotype imputation. Nat Genet. 2016;48:1279-83

15. Walter K, Min JL, Huang J, Crooks L, Memari Y, McCarthy S, et al. The UK10K project identifies rare variants in health and disease. Nature. 2015;526:82-9.

16. Bycroft C, Freeman C, Petkova D, Band G, Elliott LT, Sharp K, et al. The UK biobank resource with deep phenotyping and genomic data. Nature. 2018;562:203-9.

17. Korb CA, Kottler UB, Wolfram C, Hoehn R, Schulz A, Zwiener I, et al. Prevalence of age-related macular degeneration in a large European cohort: results from the population-based Gutenberg health study. Graefe's arch. Clin. Exp. Ophthalmol. Springer. Berlin Heidelberg. 2014; 252:1403-11.

18. Ferris FL, Wilkinson CP, Bird A, Chakravarthy U, Chew E, Csaky K, et al. Clinical classification of age-related macular degeneration. Ophthalmology. 2013;120:844-51.

19. Brandl C, Breinlich V, Stark KJ, Enzinger S, Aßenmacher M, Olden M, et al. Features of Age-Related Macular Degeneration in the General Adults and Their Dependency on Age, Sex, and Smoking: Results from the German KORA Study. Thatcher TH, editor. PLoS One. Public Libr Sci. 2016;11:e0167181.

20. Grassmann F, Mengelkamp J, Brandl C, Harsch S, Zimmermann ME, Linkohr $B$, et al. A deep learning algorithm for prediction of age-related eye disease study severity scale for age-related macular degeneration from color fundus photography. Ophthalmology. 2018;125:1410-20

21. Guenther F, Brandl C, Winkler TW, et al. Chances and challenges of machine learning-based disease classification in genetic association studies illustrated on age-related macular degeneration. Genet Epidemiol. 2020;10.1002/gepi.22336

22. De Raadt A, Warrens MJ, Bosker RJ, HAL K. Kappa Coefficients for Missing Data. Educ. Psychol. Meas. 2019.

23. Zhan X, Hu Y, Li B, Abecasis GR, Liu DJ. RVTESTS: an efficient and comprehensive tool for rare variant association analysis using sequence data. Bioinformatics. 2016;32:1423-6.

24. Gorski M, Günther F, Winkler TW, Weber BHF, Heid IM. On the differences between mega- and meta-imputation and analysis exemplified on the genetics of age-related macular degeneration. Genet Epidemiol. 2019:43:559-76.

25. Winkler TW, Day FR, Croteau-Chonka DC, Wood AR, Locke AE, Mägi R, et al. Quality control and conduct of genome-wide association meta-analyses. Nat Protoc. 2014;9:1192-212.

26. Devlin AB, Roeder K, Devlin B. Genomic Control for Association. 2013:55:997-1004.

27. Willer CJ, Li Y, Abecasis GR. METAL: fast and efficient meta-analysis of genomewide association scans. Bioinformatics. 2010;26:2190-1.

28. Harrer M, Cuijpers P, Furukawa T, Ebert DD. Doing meta-analysis in R: a hands-on guide. Lab: Prot; 2019.

29. Higgins JPT, Thomas J, Chandler J, Cumpston M, Li T, Page MJ, Welch VA (editors). Cochrane Handbook for Systematic Reviews of Interventions. 2nd Edition. Chichester: Wiley; 2019.

30. Huedo-Medina TB, Sánchez-Meca J, Marín-Martínez F, Botella J. Assessing heterogeneity in meta-analysis: Q statistic or 12 index? Psychol Methods. 2006;11:193-206

31. Yang J, Ferreira T, Morris AP, Medland SE, Madden PAF, Heath AC, et al. Conditional and joint multiple-SNP analysis of GWAS summary statistics identifies additional variants influencing complex traits. Nat Genet Nature Publishing Group. 2012;44:369-75.

32. Kichaev G, Yang WY, Lindstrom S, Hormozdiari F, Eskin E, Price AL, et al. Integrating functional data to prioritize causal variants in statistical finemapping studies. PLoS Genet. 2014;10.

33. McLaren W, Gil L, Hunt SE, Riat HS, Ritchie GRS, Thormann A, et al. The Ensembl Variant Effect Predictor. Genome Biol. 2016;17:122.

34. Ratnapriya R, Sosina OA, Starostik MR, Kwicklis M, Kapphahn RJ, Fritsche LG, et al. Retinal transcriptome and eQTL analyses identify genes associated with age-related macular degeneration. Nat Genet. 2019;51:606-10.

35. Aguet F, Brown AA, Castel SE, Davis JR, He Y, Jo B, et al. Genetic effects on gene expression across human tissues. Nature. 2017;550:204-13.

36. Hormozdiari F, van de Bunt M, Segrè AV, Li X, Joo JWJ, Bilow M, et al. Colocalization of GWAS and eQTL signals detects target genes. Am. J Hum Genet. 2016;99:1245-60.

37. Bryan JM, Fufa TD, Bharti K, Brooks BP, Hufnagel RB, McGaughey DM. Identifying core biological processes distinguishing human eye tissues with precise systems-level gene expression analyses and weighted correlation networks. Hum Mol Genet Narnia. 2018:27:3325-39.

38. Bult CJ, Blake JA, Smith CL, Kadin JA, Richardson JE, Anagnostopoulos A, et al. Mouse genome database (MGD) 2019. Nucleic Acids Res Oxford University Press. 2019;47:D801-6.

39. Grassmann F, Kiel C, Zimmermann ME, Gorski M, Grassmann V, Stark K, et al. Genetic pleiotropy between age-related macular degeneration and 16 complex diseases and traits. Genome Med Genome Medicine. 2017:9:1-13.

40. MacArthur J, Bowler E, Cerezo M, Gil L, Hall P, Hastings E, et al. The new NHGRI-EBI catalog of published genome-wide association studies (GWAS catalog). Nucleic Acids Res. 2017;45:D896-901.

41. Beck T, Hastings RK, Gollapudi S, Free RC, Brookes AJ. GWAS central: a comprehensive resource for the comparison and interrogation of genome-wide association studies. Eur J Hum Genet Nature Publishing Group. 2014;22:949-52.

42. Canela-Xandri O, Rawlik K, Tenesa A. An atlas of genetic associations in UK biobank. Nat Genet Springer US. 2018;50:1593-9.

43. oav B, Hochberg Y. Controlling the False Discovery Rate - a Practical and Powerful Approach to Multiple Testing. Journal of the Royal Statistical Society Series B-Methodological 1995.pdf. JR Stat Soc Ser B. 1995.

44. Winkler TW, Brandl C, Grassmann F, Gorski M, Stark K, Loss J, et al. Investigating the modulation of genetic effects on late AMD by age and sex: lessons learned and two additional loci. PLoS One. 2018;13:1-21.

45. Bulik-Sullivan B, Loh PR, Finucane HK, Ripke S, Yang J, Patterson N, et al. LD score regression distinguishes confounding from polygenicity in genome-wide association studies. NatGenet Nature Publishing Group. 2015;47:291-5.

46. Winkler TW, Günther F, Höllerer S, Zimmermann M, Loos RJ, Kutalik Z, et al. A joint view on genetic variants for adiposity differentiates subtypes with distinct metabolic implications. Nat Commun Springer US. 2018;9

47. Kuleshov MV, Jones MR, Rouillard AD, Fernandez NF, Duan Q, Wang Z, et al. Enrichr: a comprehensive gene set enrichment analysis web server 2016 update. Nucleic Acids Res. 2016:44:W90-7.

48. Gao X, Starmer J, Martin ER. A multiple testing correction method for genetic association studies using correlated single nucleotide polymorphisms. Genet Epidemiol. 2008.

49. Fabregat $A$, Jupe $S$, Matthews $L$, Sidiropoulos K, Gillespie M, Garapati $P$, et al. The Reactome Pathway Knowledgebase. Nucleic Acids Res. 2018; 46:D649-55.

50. Hageman GS, Luthert PJ, Victor Chong NH, Johnson LV, Anderson DH, Mullins RF. An integrated hypothesis that considers drusen as biomarkers of immune-mediated processes at the RPE-Bruch's membrane interface in aging and age-related macular degeneration. Prog Retin Eye Res. 2001;20:705-32.

51. Johnson LV, Leitner WP, Staples MK, Anderson DH. Complement activation and inflammatory processes in Drusen formation and age related macular degeneration. Exp Eye Res. 2001;73:887-96.

52. Vogt SD, Curcio CA, Wang L, Li CM, McGwin G Jr, Medeiros NE, et al. Retinal pigment epithelial expression of complement regulator CD46 is altered early in the course of geographic atrophy. Exp Eye Res. 2011;93: 413-23.

53. Seya $T$, Atkinson JP. Functional properties of membrane cofactor protein of complement. Biochem J. 1989;264:581-8.

54. Cattaneo R. Four viruses, two Bacteria, and one receptor: membrane cofactor protein (CD46) as pathogens' magnet. J Virol. 2004;78:4385-8

55. Cardone J, Le Friec G, Kemper C. CD46 in innate and adaptive immunity: an update. Clin Exp Immunol. 2011;164:301-11.

56. Haralambieva IH, Ovsyannikova IG, Kennedy RB, Larrabee BR, Zimmermann MT, Grill DE, et al. Genome-wide associations of CD46 and IFI44L genetic variants with neutralizing antibody response to measles vaccine. Hum Genet. 2017:136:421-35.

57. Lewis RA. Oculocutaneous Albinism Type 1 [Internet]. GeneReviews ${ }^{\oplus}$. Seattle: University of Washington; 1993.

58. Galván-Femenía I, Obón-Santacana M, Piñeyro D, Guindo-Martinez M, Duran $X$, Carreras A, et al. Multitrait genome association analysis identifies new susceptibility genes for human anthropometric variation in the GCAT cohort. J Med Genet. 2018:765-78.

59. Stokowski RP, Pant PVK, Dadd T, Fereday A, Hinds DA, Jarman C, et al. A Genomewide association study of skin pigmentation in a south Asian population. Am J Hum Genet Cell Press. 2007;81:1119-32. 
60. Gao XR, Huang H, Kim H. Genome-wide association analyses identify 139 loci associated with macular thickness in the UK biobank cohort. Hum Mol Genet. 2019;28:1162-72.

61. Brandl C, Brücklmayer C, Günther F, Zimmermann ME, Küchenhoff H, Helbig $\mathrm{H}$, et al. Retinal layer thicknesses in early age-related macular degeneration: results from the German AugUR study. Invest Ophthalmol Vis Sci. 2019;60: 1581-94.

\section{Publisher's Note}

Springer Nature remains neutral with regard to jurisdictional claims in published maps and institutional affiliations.

Ready to submit your research? Choose BMC and benefit from:

- fast, convenient online submission

- thorough peer review by experienced researchers in your field

- rapid publication on acceptance

- support for research data, including large and complex data types

- gold Open Access which fosters wider collaboration and increased citations

- maximum visibility for your research: over $100 \mathrm{M}$ website views per year

At $\mathrm{BMC}$, research is always in progress.

Learn more biomedcentral.com/submissions 